\title{
QUELQUES DONNÉES \\ SUR LES VARIATIONS SAISONNIËRES \\ DU PLANCTON ET \\ SUR LES GARACTÉRISTIQUES HYDROLOGIQUES \\ EN MER DU NORD, \\ AU LARGE D'OSTENDE ET DE NIEUPORT. \\ PÉRIODE 1969-1971
}

\author{
par Rosine Segebarth-ORBan (*) \\ Laboratoire d'Anatomie Comparée \\ (Prof. J. BouLLLON) \\ Université Libre de Bruxelles
}

\section{RÉSUMÉ}

L'article ci-dessous contient des observations sur le milieu marin, faites de mars 1969 à mars 1971, à quelques stations au large de Nieuport et d'Ostende (Belgique).

Dans la première partie se trouve un rappel des caractéristiques générales de la météorologie et des courants ainsi qu'une description des variations saisonnières de la salinité et de la concentration de quelques sels minéraux (phosphates, silicates, nitrates, nitrites et azote ammoniacal).

La seconde partie décrit globalement les variations de la fréquence des principaux groupes du zooplancton.

Pour les différents domaines envisagés, les stations étudiées suivent les mêmes courbes de fluctuation. La plupart des phénomènes observés semblent dûs à des apports d'eaux douces côtières ; en effet, la dessalure. la concentration en éléments nutritifs et la quantité de phytoplancton diminuent lorsqu'on s'éloigne de la côte.

\section{INTRODUCTION}

Les premières observations sur le zooplancton des eaux littorales belges ont été faites par G. GILson (1900 et 1907). Les travaux ultérieurs concernent principalement les estuaires et les ports du littoral comme Ostende (E. Leloup, L. VAN Mees et S. Lefevere, 1956 ; N. De Pauw, 1969), Nieuport (M. C. GeRETS, 1970) et le Bas-Escaut (E. Leloup et B. Konietzko, 1956).

(*) Bénéficiaire d'une bourse de spécialisation de l'Institut pour l'encouragement de la Recherche Scientifique dans l'Industrie et l'Agriculture (I.R.S.I.A.) de 1969 à 1971 . 
Au cours de ce travail, nous avons tenté de décrire globalement les variations saisonnières du zooplancton à quelques stations au large de Nieuport et d'Ostende.

Ces variations sont profondément influencées par les caractéristiques physico-chimiques du milieu marin et par l'abondance du phytoplancton. C'est pourquoi la première partie de cette étude traite des observations météorologiques et hydrologiques, et de quelques données quantitatives concernant le phytoplancton.

Les prélèvements ont été effectués pendant deux ans : de mars 1969 à mars 1971.

Depuis 1971, la Commission Interministérielle belge de la Politique Scientifique (C.I.P.S.) a entrepris en Mer du Nord un vaste "Programme national sur l'environnement physique et biologique (Pollution des eaux - projet Mer) ». Ce travail de recherches pluridisciplinaires se poursuit encore actuellement et prend des mesures continues à différents points d'un réseau couvrant l'ensemble de la Mer du Nord. Toutefois, les stations que nous avons étudiées (voir chap. 2 et fig. 1 ) sont très côtières et en dehors des mailles de ce réseau.

\section{MATÉRIEL ET MÉTHODES}

Au cours de la première année (1969-1970), des échantillons furent prélevés tous les quinze jours dans les eaux de surface des cinq endroits suivants :

TABLEAU I (cfr fig. 1)

\begin{tabular}{l|c|c|c|c}
\hline \multicolumn{1}{c|}{ Stations } & $\begin{array}{c}\text { Latitude } \\
\text { Nord }\end{array}$ & $\begin{array}{c}\text { Longitude } \\
\text { Est }\end{array}$ & $\begin{array}{c}\text { Hauteur } \\
\text { d'eau } \\
\text { aux basses } \\
\text { mers de } \\
\text { vive-eau } \\
(\mathrm{m})\end{array}$ & $\begin{array}{c}\text { Distance } \\
\text { en milles } \\
\text { de la } \\
\text { côte }\end{array}$ \\
\hline & & & & \\
\hline $\begin{array}{l}\text { 1. Nieuwpoortbank } \\
\text { 2. Oost-Dijek }\end{array}$ & $51^{\circ} 10^{\prime} 25^{\prime \prime}$ & $2^{\circ} 36^{\prime} 05^{\prime \prime}$ & 6 & 3 \\
3. Akkaert & $51^{\circ} 21^{\prime} 40^{\prime \prime}$ & $2^{\circ} 31^{\prime} 10^{\prime \prime}$ & 10 & 14 \\
4. Oostendebank-Oost & $51^{\circ} 23^{\prime} 40^{\prime \prime}$ & $2^{\circ} 52^{\prime} 00^{\prime \prime}$ & 10 & 8,5 \\
$51^{\circ} 17^{\prime} 25^{\prime \prime}$ & $2^{\circ} 51^{\prime} 55^{\prime \prime}$ & 7 & 3,5 \\
5. Buitenstroombank & $51^{\circ} 15^{\prime} 15^{\prime \prime}$ & $2^{\circ} 51^{\prime} 45^{\prime \prime}$ & 5 & 2
\end{tabular}


Les mesures envisagées ont été les suivantes : température, salinité et dosage de la chlorophylle « a » dans les eaux de surface.

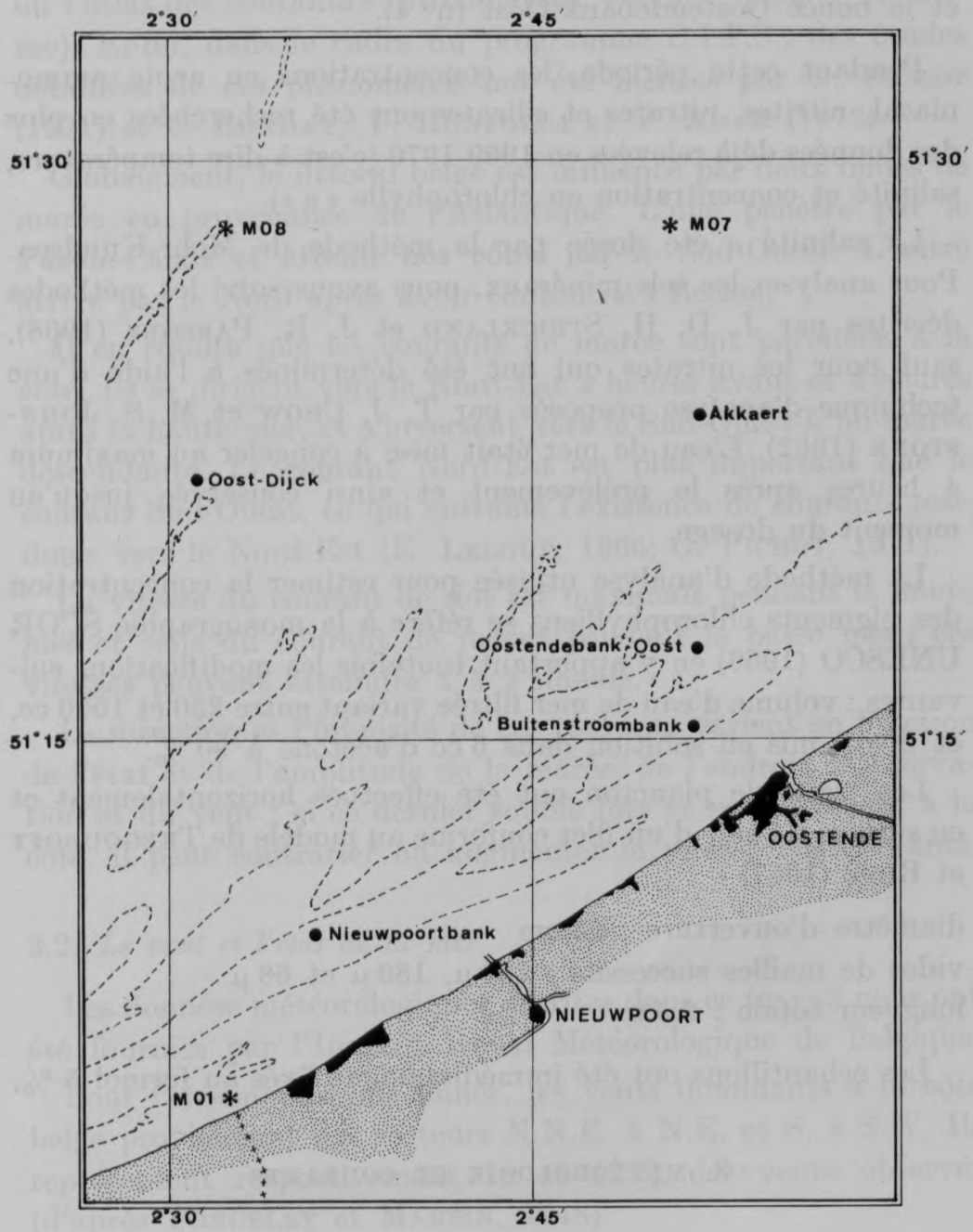

Fig. 1. - Stations de prélèvements (d'après la carte des "Banes des Flandres" du service Hydrographique de la Côte belge). Isobathes : ...: $8 \mathrm{~m}$. 
Au cours de la deuxième année (1970-1971) des échantillons furent pris en surface tous les 10 jours, à 2 stations :

la bouée Akkaert ( $\mathrm{n}^{\circ} 3$ )

et la bouée Oostendebank-Oost ( $\left.n^{0} 4\right)$.

Pendant cette période, les concentrations en azote ammoniacal, nitrites, nitrates et silicates ont été recherchées en plus des données déjà relevées en 1969-1970 (c'est-à-dire température, salinité et concentration en chlorophylle «a»).

La salinité a été dosée par la méthode de Mohr-Knudsen. Pour analyser les sels minéraux, nous avons suivi les méthodes décrites par J. D. H. Strickland et J. R. Parsons (1968), sauf pour les nitrates qui ont été déterminés à l'aide d'une technique d'analyse proposée par T. J. Cноw et M. S. JoнNSTONE (1962). L'eau de mer était mise à congeler au maximum 4 heures après le prélèvement et ainsi conservée jusqu'au moment du dosage.

La méthode d'analyse utilisée pour estimer la concentration des pigments chlorophylliens se réfère à la monographie SCOR UNESCO (1966) en y apportant toutefois les modifications suivantes : volume d'eau de mer filtrée variant entre 250 et $1000 \mathrm{cc}$, et filtres mis en solution dans $6 \mathrm{cc}$ d'acétone à $90 \%$.

Les traits de plancton ont été effectués horizontalement et en surface à l'aide d'un filet conforme au modèle de TREGOUBOFF et Rose (1957) :

diamètre d'ouverture : $65 \mathrm{~cm}$

vides de mailles successifs : $280 \mu, 180 \mu$ et $68 \mu$

longueur totale : $2,5 \mathrm{~m}$.

Les échantillons ont été immédiatement fixés au formol $5 \%$.

\section{MÉTÉOROLOGIE ET COURANTS}

\subsection{Courants}

Les courants jouent un rôle capital dans le transport des organismes et des matières en suspension, mais l'étude de ce facteur dépasse le cadre de notre travail. 
En Mer du Nord, ce problème a été abordé par G. GILSON (1924) et E. Leloup (1966).

Le Service Hydrographique de la côte belge a édité en 1969 un "atlas des courants" (Stroomatlas, Vlaamse banken, Noordzee). Enfin, dans le cadre du programme C.I.P.S., des études détaillées de ces phénomènes ont été menées par G. Pichot (1971) et C. Ronday, Y. Runfolla et Y. Adam (1972).

Globalement, le littoral belge est influencé par deux ondes de marée en provenance de l'Atlantique. L'une pénètre par le Pas-de-Calais et atteint nos côtes par le Sud-Ouest. L'autre arrive par le Nord après avoir contourné l'Écosse.

Il en résulte que les courants de marée sont parallèles à la côte. Ils se dirigent vers le Nord-Est 2 heures avant et 3 heures après la haute mer, et s'inversent vers le Sud-Ouest à mi-marée descendante. Le courant Nord-Est est plus important que le courant Sud-Ouest, ce qui entraîne l'existence de courants résiduels vers le Nord-Est (E. Leloup, 1966; G. Ріснот, 1971).

La vitesse $d u$ courant de flot est maximale pendant la haute mer et celle du courant de jusant pendant la basse mer ; ces vitesses peuvent atteindre 2 à 3 nœuds.

La direction et l'intensité de ces courants varient en fonction de l'état et de l'amplitude de la marée, de l'endroit d'observation et du vent : si ce dernier souffie fort et parallèlement à la côte, il peut contrarier ou augmenter la vitesse des courants.

\subsection{Le vent et l'état de la mer}

Les données météorologiques décrites dans ce travail nous ont été fournies par l'Institut Royal Météorologique de Belgique.

Pour l'ensemble d'une année, les vents dominants à la côte belge proviennent des secteurs N.N.E. à N.E. et S. à S.W. Ils représentent respectivement 18 et $47 \%$ des vents observés (d'après Poncelet et Martin, 1948).

Sous l'action des courants résiduels et des vents dominants du S.W., nos eaux côtières se déplacent vers le N.E. (G. GILson, 1900 et 1924 ; E. LeLoup, 1966).

L'état de la mer varie en fonction de la force et de la direc- 
tion du vent; si les vents viennent du large (N.N.W.) la mer est beaucoup plus agitée que s'ils viennent de la côte (S.S.E.).

De mars 1969 à mars 1971, la moyenne de l'état de la mer par décade fut rarement inférieure à force 2 ; le brassage des eaux a donc été fréquent, permettant une mise en suspension régulière des sédiments aux endroits de faible profondeur; ce qui est en particulier le cas des stations que nous avons étudiées (cfr. hauteur d'eau-tableau I).

\subsection{Température de l'eau}

Au cours des deux années d'observations, la température de l'eau de surface s'est élevée progressivement depuis le mois de mars, quasi simultanément avec la température de l'air (figures 2 et 3). La température de l'eau passe par un maximum vers la

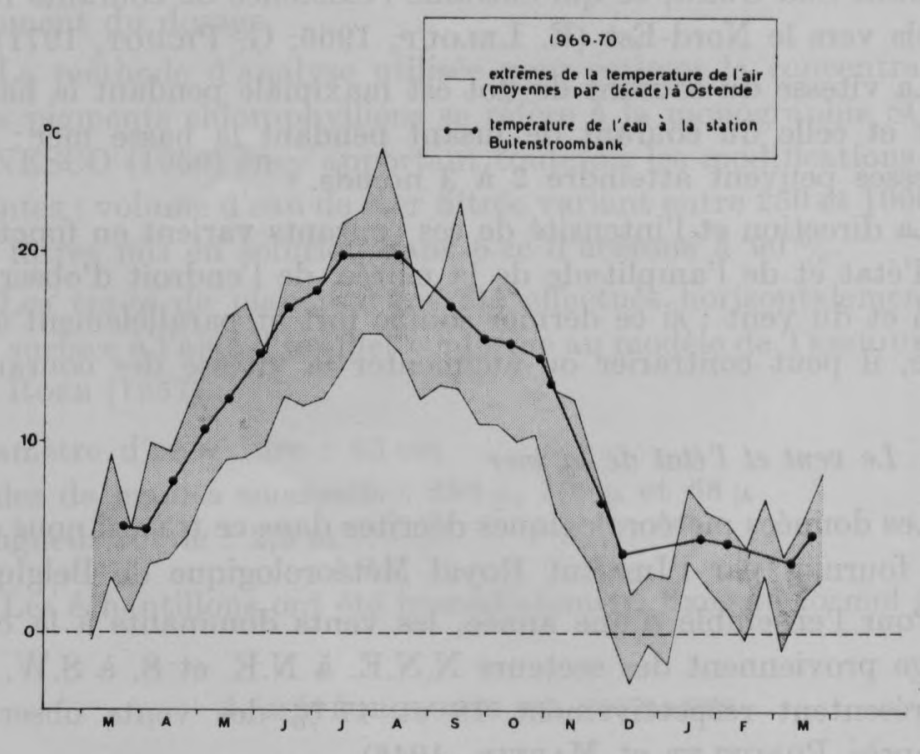

Fig. 2

fin juillet (environ $18,5^{\circ} \mathrm{C}$ ). A partir de ce moment, elle commence à se refroidir et atteint ses valeurs minimales pendant les mois de janvier, février et mars. 


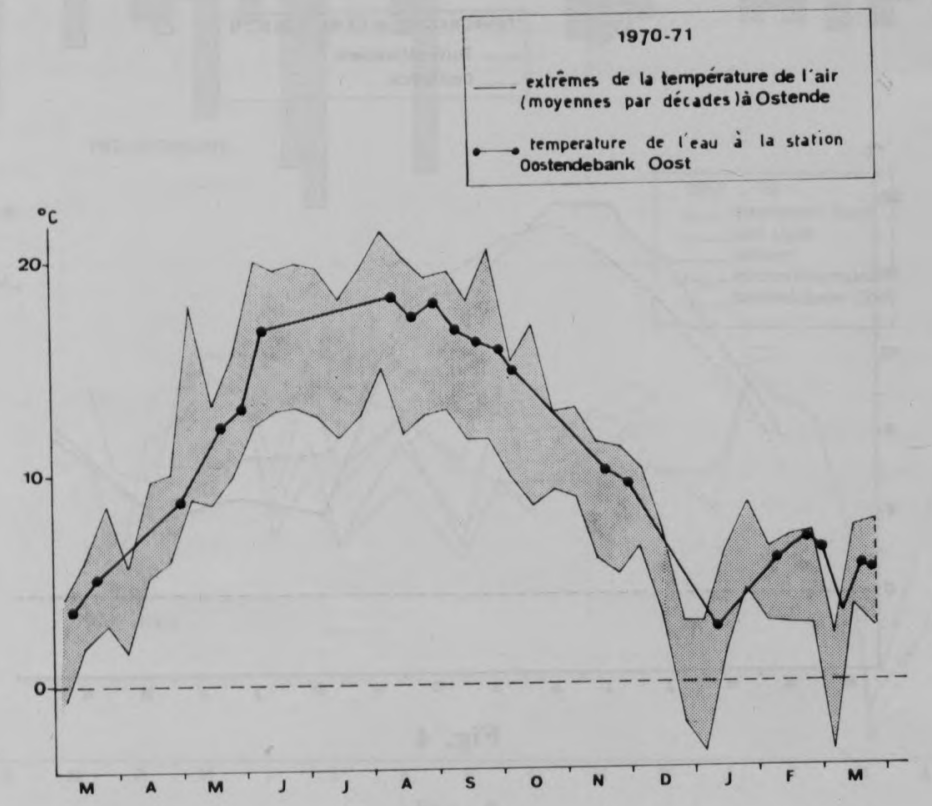

Fig. 3

Aux stations oost-Dijck et Nieuwpoortbank, l'eau est plus froide en été et plus chaude en hiver qu'à la Buitenstroombank située plus à l'Est et plus près de la côte (figures 4 et 5).

Ces résultats, ainsi que des renseignements sur la température de l'eau entre les musoirs de l'estacade d'Ostende (N. De Pauw 1969), amènent à penser que l'inertie thermique augmente lorsqu'on s'éloigne vers le large et vers le Sud-Ouest.

Cependant, les différences observées entre les bouées Nieuwpoortbank et Buitenstroombank excèdent rarement $0,5^{\circ} \mathrm{C}$. Il faudrait dès lors effectuer des prélèvements simultanés aux deux stations pour vérifier si ces différences ne sont pas imputables aux variations diurnes de la température de l'eau.

\subsection{Salinité}

Les figures 6 et 7 représentent les précipitations et les variations de salinité observées en 1969-1970 et 1970-1971. 


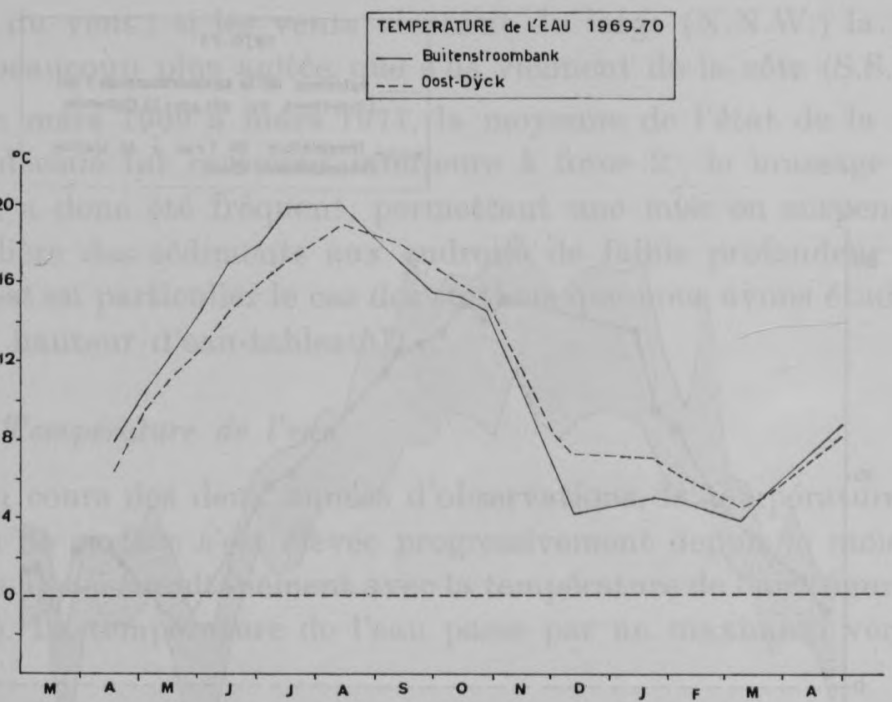

Fig. 4

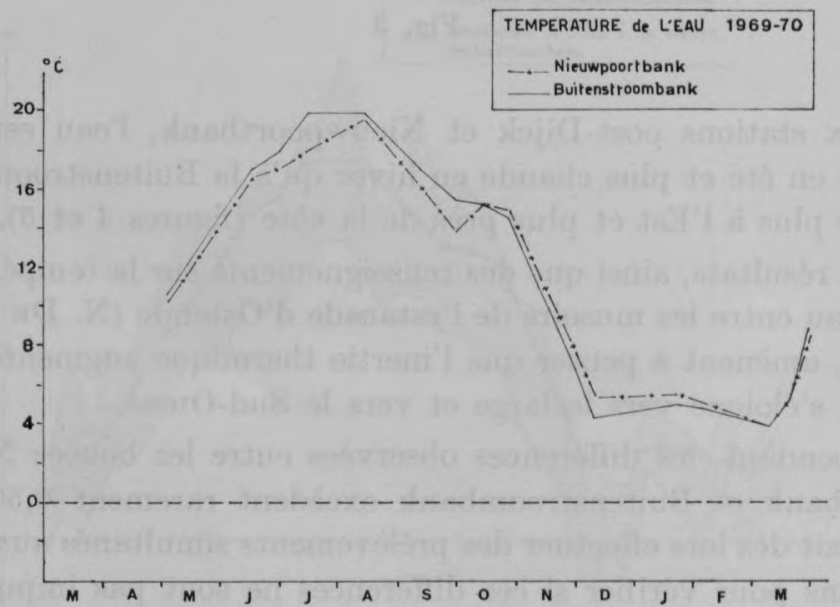

Fig. 5

Dans l'ensemble, les stations suivent les mêmes courbes de fluctuation.

Mais à partir des moyennes annuelles, on peut faire les constatations suivantes (cfr. tableau II et graphique 6). 


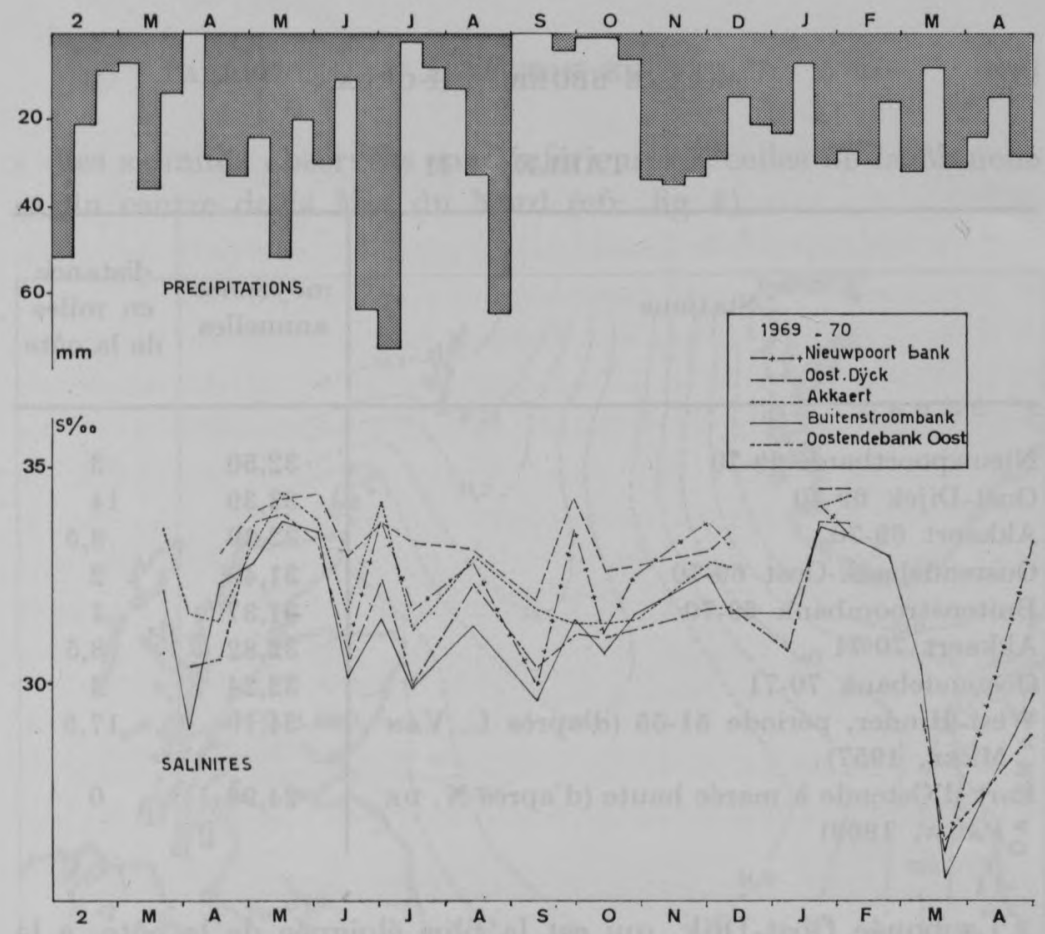

Fig. 6

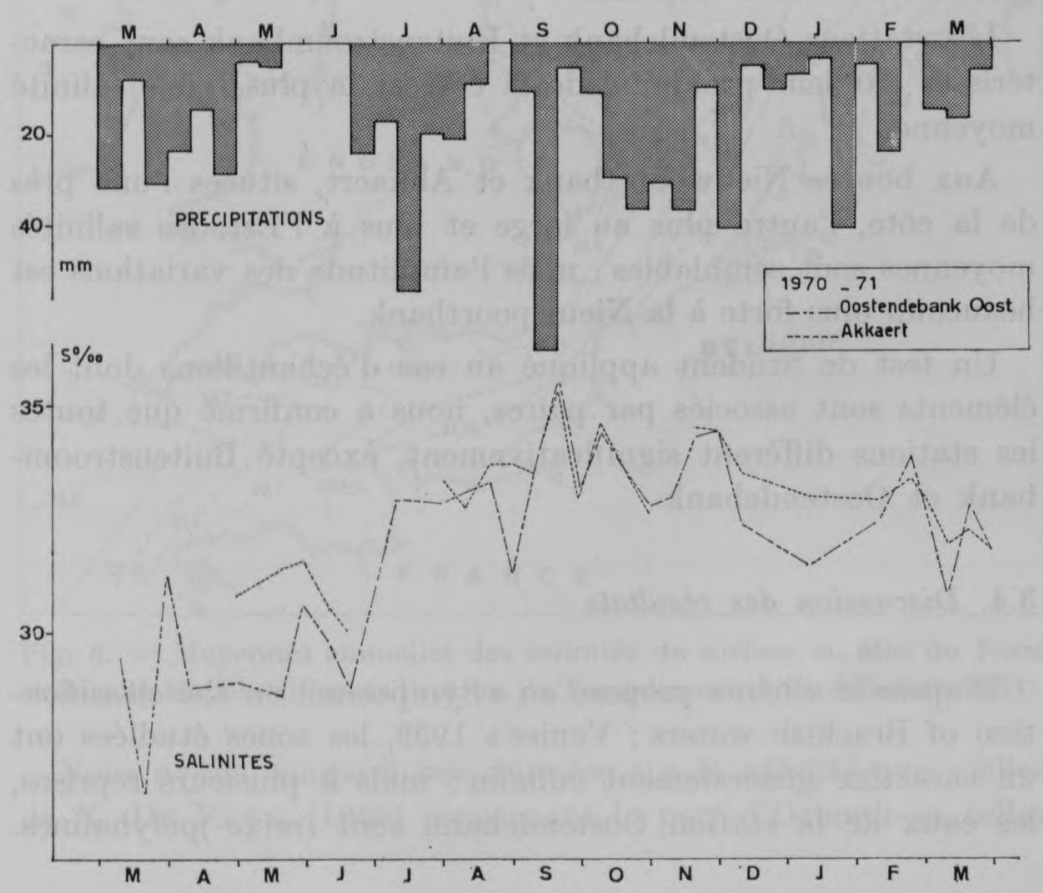

Fig. 7 


\begin{tabular}{l|c|c}
\hline \multicolumn{1}{c|}{ Stations } & $\begin{array}{c}\text { moyennes } \\
\text { annuelles }\end{array}$ & $\begin{array}{c}\text { distance } \\
\text { en miles } \\
\text { de la côte }\end{array}$ \\
& & \\
\hline & & \\
Nieuwpoortbank 69-70 & 32,50 & 3 \\
Oost-Dijek 69-70 & 33,39 & 14 \\
Akkaert 69-70 & 32,39 & 8,5 \\
Oostendebank-Oost 69-70 & 31,37 & 2 \\
Buitenstroombank 69-70 & 32,82 & 8,5 \\
Akkaert 70-71 & 32,24 & 2 \\
Oostendebank 70-71 & 34,19 & 17,5 \\
West-Hinder, période 51-55 (d'après L. VAN & & \\
MEEL, 1957) & 24,98 & 0 \\
Port d'Ostende à marée haute (d'après N. DE & & \\
PAUw, 1969) &
\end{tabular}

La bouée Oost-Dijk, qui est la plus éloignée de la côte, a la plus haute salinité moyenne.

Les stations Oostendebank et Buitenstroombank sont caractérisées par leur proximité de la côte et la plus faible salinité moyenne.

Aux bouées Nieuwpoortbank et Akkaert, situées l'une près de la côte, l'autre plus au large et plus à l'Est, les salinités moyennes sont semblables; mais l'amplitude des variations est beaucoup plus forte à la Nieuwpoortbank.

Un test de Student appliqué au cas d'échantillons dont les éléments sont associés par paires, nous a confirmé que toutes les stations diffèrent significativement, excepté Buitenstroombank et Oostendebank.

\subsection{Discussion des résultats}

D'après le schéma proposé au "Symposium on the classification of Brackish waters ; Venise " 1959, les zones étudiées ont un caractère généralement euhalin ; mais à plusieurs reprises, les eaux de la station Oostendebank sont (mixo-)polyhalines. 
Les salinités observées sont inférieures à celles de la Manche et du centre de la Mer du Nord (cfr. fig. 8).

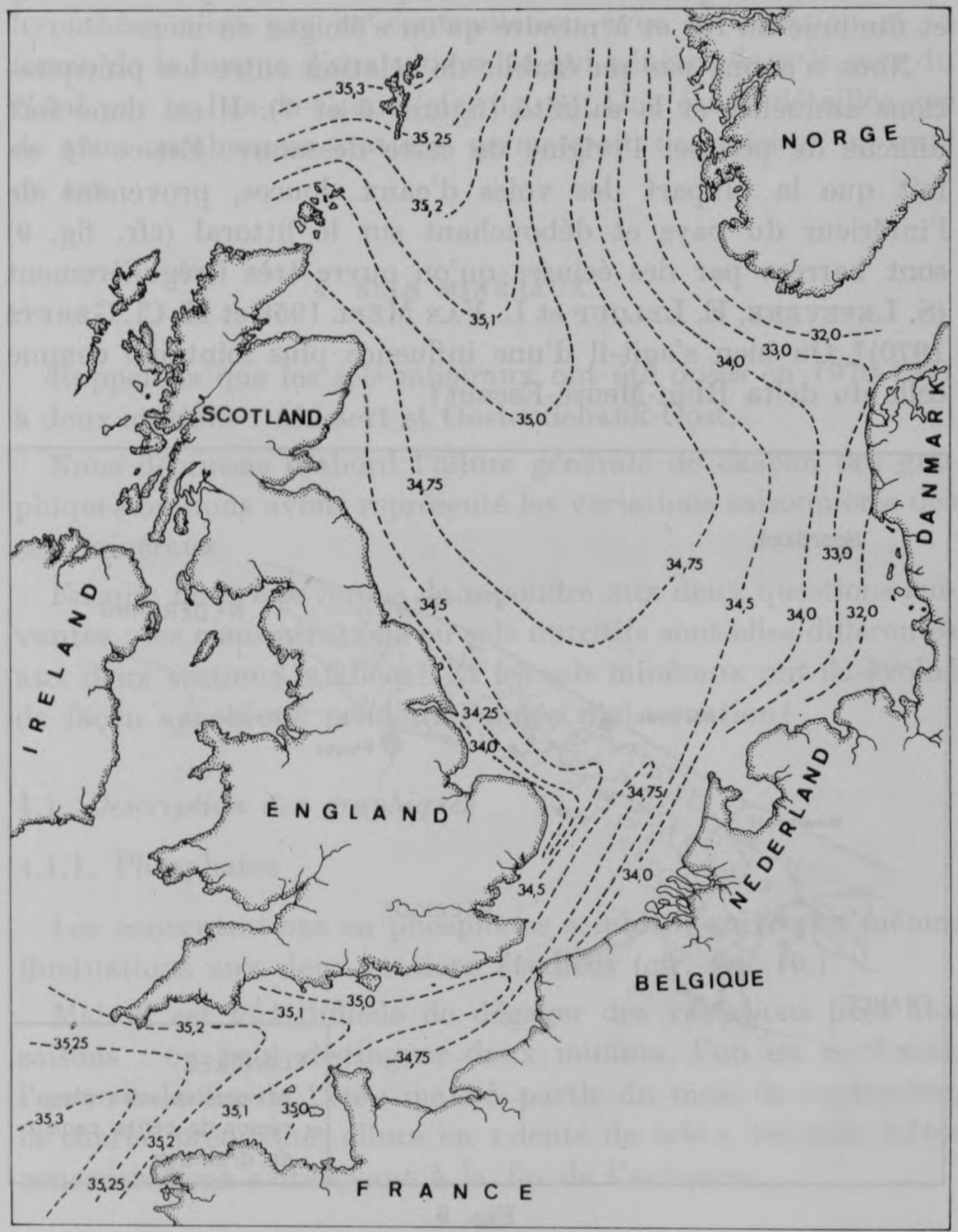

Fig. 8. - Moyennes annuelles des salinités de surface en Mer du Nord et dans la Manche (d'après l'"Atlas de Température et de Salinité " 1933).

Nous avons comparé nos données sur la salinité avec celles de N. DE Pauw (1969) concernant le port d'Ostende et celles 
de L. VAN MeeL (1957) au bateau-phare West-Hinder, situé à la limite des eaux territoriales (cfr. tableau II).

Il en ressort que la dessalure est plus accusée près des côtes et diminue au fur et à mesure qu'on s'éloigne en mer.

Nous n'avons pas pu établir de relation entre les précipitations annuelles et la salinité (figures 6 et 7 ). Il est done fort difficile de préciser l'origine de cette dessalure. Est-ce dû au fait que la plupart des voies d'eaux douces, provenant de l'intérieur du pays et débouchant sur le littoral (efr. fig. 9) sont barrées par des écluses qu'on ouvre très irrégulièrement (S. Lefevere, E. Leloup et L. Van Meel 1956 et M. Cl. Gerets 1970)? Ou bien s'agit-il d'une influence plus lointaine comme celle du delta Rhin-Meuse-Escaut?

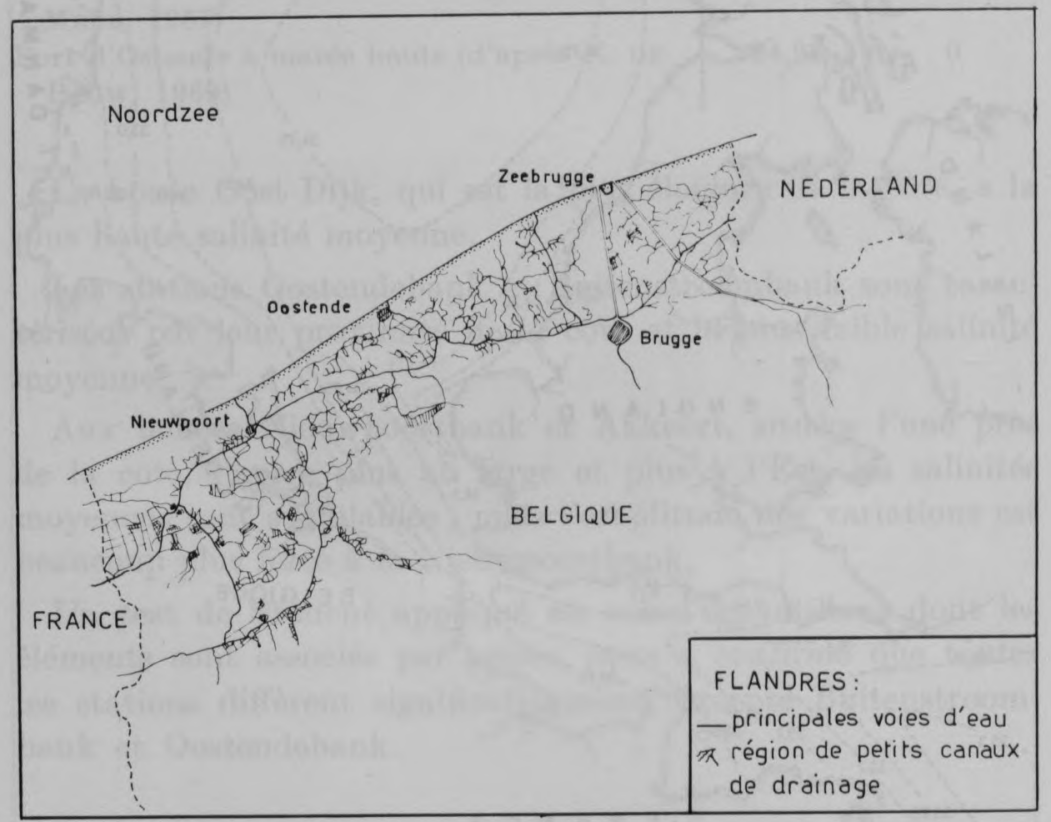

Fig. 9

Les caractéristiques de la Nieuwpoortbank appellent quelques remarques : d'une part, l'amplitude des variations y est forte, ce qui pourrait être dû à des apports d'eaux douces de l'Yser ; d'autre part sa salinité moyenne est plus élevée qu'aux stations 
Oostendebank et Buitenstroombank situées à même distance de la côte. Ceci nous amène à soupçonner l'existence d'un deuxième gradient croissant de salinité vers l'Ouest de la côte belge. Cette hypothèse n'est pas en contradiction avec la direction et la forme de la langue d'eau plus salée qui pénètre dans la mer du Nord par le Pas-de-Calais (cfr. fig. 8). Une étude détaillée sur de plus nombreuses stations permettrait de préciser ce qu'il en est.

\section{SELS MINÉRAUX}

Rappelons que les sels minéraux ont été dosés en 1970-1971 à deux stations : Akkaert et Oostendebank-Oost.

Nous décrirons d'abord l'allure générale de chacun des graphiques où nous avons représenté les variations saisonnières des sels minéraux.

Ensuite nous essayerons de répondre aux deux questions suivantes : les concentrations en sels nutritifs sont-elles différentes aux deux stations étudiées? Et les sels minéraux ont-ils évolué de façon synchrone pendant l'année d'observation?

\subsection{Description des graphiques}

\subsubsection{Phosphates}

Les concentrations en phosphates semblent suivre les mêmes fluctuations aux deux stations étudiées (cfr. fig. 10.)

Mais il est fort difficile de dégager des variations liées aux saisons : on peut distinguer deux minima, l'un en avril-mai, l'autre à la fin de l'été ; mais à partir du mois de septembre, la courbe prend une allure en "dents de scie", les plus fortes concentrations s'observant à la fin de l'automne.

\subsubsection{Silicates}

Les plus fortes teneurs en silice s'observent du mois d'octobre au mois d'avril, tandis qu'en été les courbes passent par un minimum (fig. 11). 


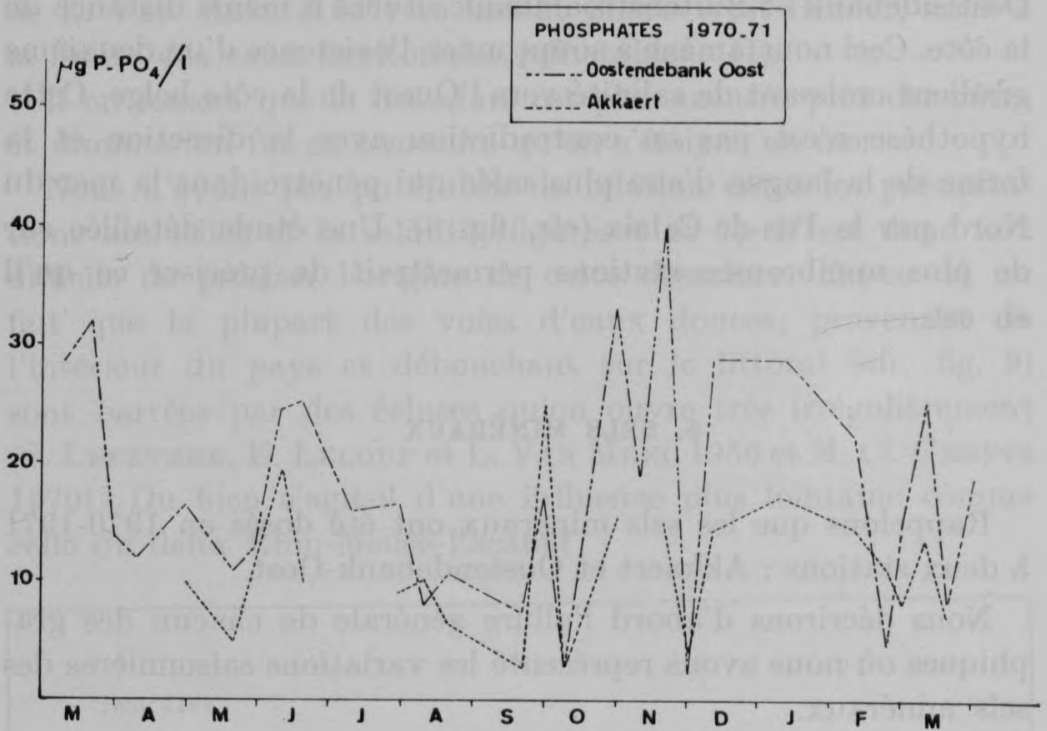

Fig. 10

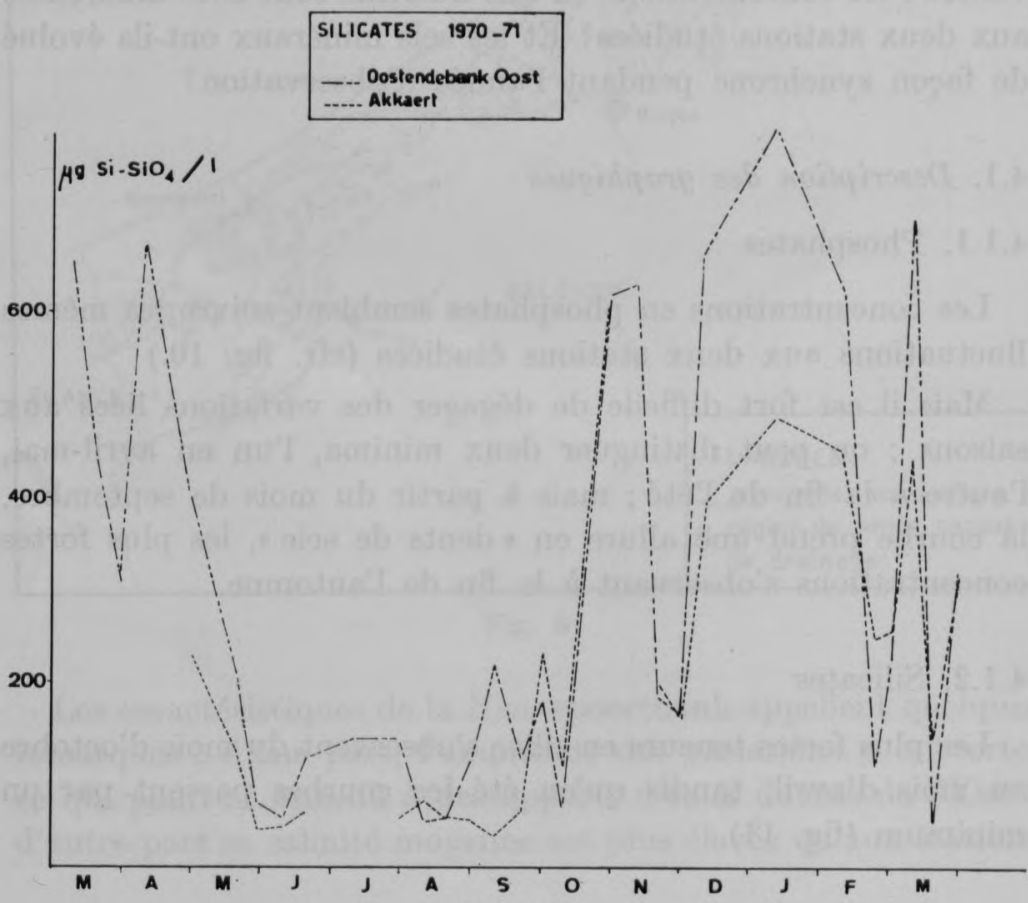

Fig. 11 


\subsubsection{Nitrates}

Dans l'ensemble, les variations observées sur la figure 12 ressemblent à celles des silicates.

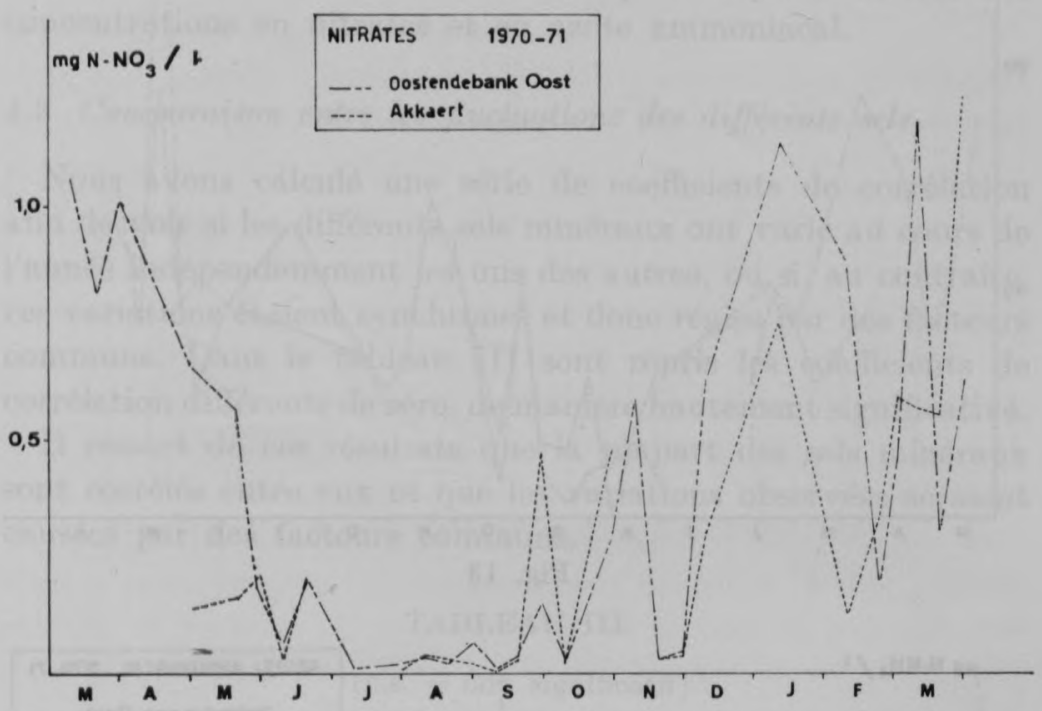

Fig. 12

\subsubsection{Nitrites}

Le taux des nitrites, important au printemps, passe par un minimum au mois d'août et recommence à augmenter jusqu'en mars où il atteint des valeurs de plus de $30 \mu \mathrm{g} \mathrm{N}-\mathrm{NO}_{2} / 1$ (fig. 13).

\subsubsection{Azote ammoniacal}

Tout au long de l'année, le taux d'azote ammoniacal oscille entre 25 et $300 \mu \mathrm{g} / 1$, sauf un pic important en janvier 1971 pour les deux stations (environ $700 \mu \mathrm{g} / 1$ ) et 3 valeurs très élevées à la bouée Akkaert (fig. 14).

\subsection{Comparaison entre les stations Akkaert et Oostendebank-Oost}

Afin de voir si les concentrations moyennes en sels minéraux diffèrent aux deux stations, nous les avons comparées entre elles à l'aide d'un test $t$ de Student pour valeurs couplées. 


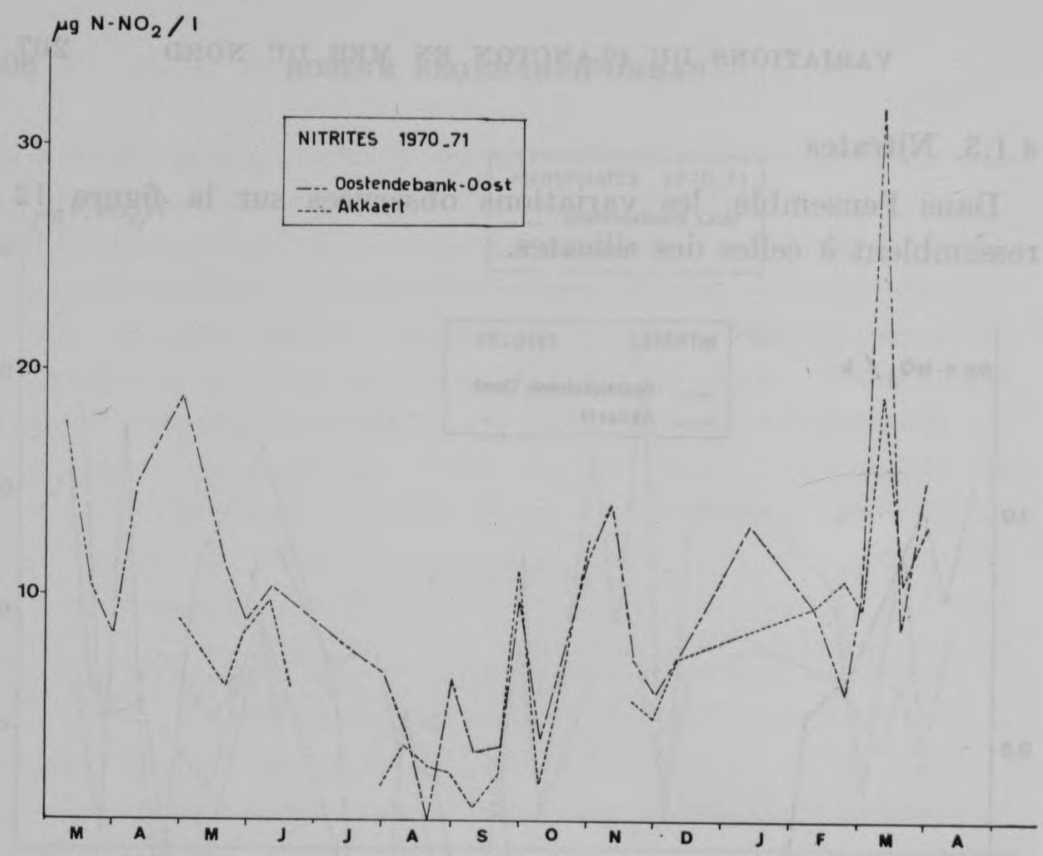

Fig. 13

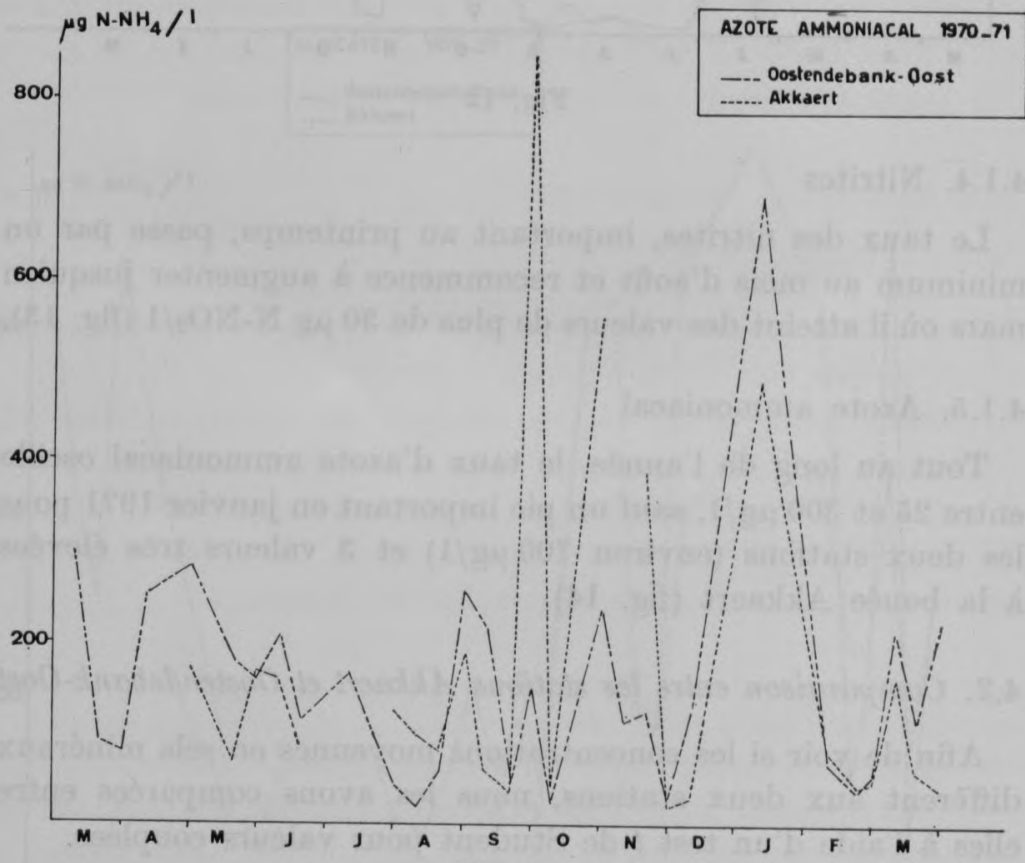

Fig. 14 
Nous avons ainsi constaté que les concentrations en phosphates, silicates et nitrites sont significativement plus élevées à la station côtière Oostendebank (avec $p=0,05$ ). Par contre, les deux stations ne se différencient pas du point de vue des concentrations en nitrates et en azote ammoniacal.

\subsection{Comparaison entre les fluctuations des différents sels}

Nous avons calculé une série de coefficients de corrélation a fin de voir si les différents sels minéraux ont varié au cours de l'année indépendemment les uns des autres, ou si, au contraire, ces variations étaient synchrones et donc régies par des facteurs communs. Dans le tableau III sont repris les coefficients de corrélation différents de zéro, de manière hautement significative.

Il ressort de ces résultats que la plupart des sels minéraux sont corrélés entre eux et que les variations observées seraient causées par des facteurs communs.

\section{TABLEAU III}

(n.s. = non significatif)

\begin{tabular}{l|c|c}
\hline & Oostendebank & Akkaert \\
\hline nitrites-nitrates & 0,78 & 0,71 \\
nitrates-silicates & 0,86 & 0,61 \\
nitrites-silicates & 0,62 & 0,68 \\
phosphates-silicates & 0,66 & n.s. \\
azote ammoniacal-silicates & 0,60 & n.s. \\
azote ammoniacal-nitrates & 0,58 & n.s. \\
phosphates-azote ammoniacal & n.s. & 0,76 \\
phosphates-nitrites & n.s. & 0,53
\end{tabular}

\subsection{Discussion des résultats}

Les plus hautes concentrations de sels minéraux se rencontrent en hiver. Mais nos graphiques prennent une allure particulière car les maxima hivernaux sont chaque fois suivis de minima et confèrent à toutes les courbes une allure en "dents de scie».

Or, dans la plupart des cas, les augmentations de la concen- 
tration en éléments nutritifs correspondent à une diminution de la salinité (cfr. en exemple, la figure 15).

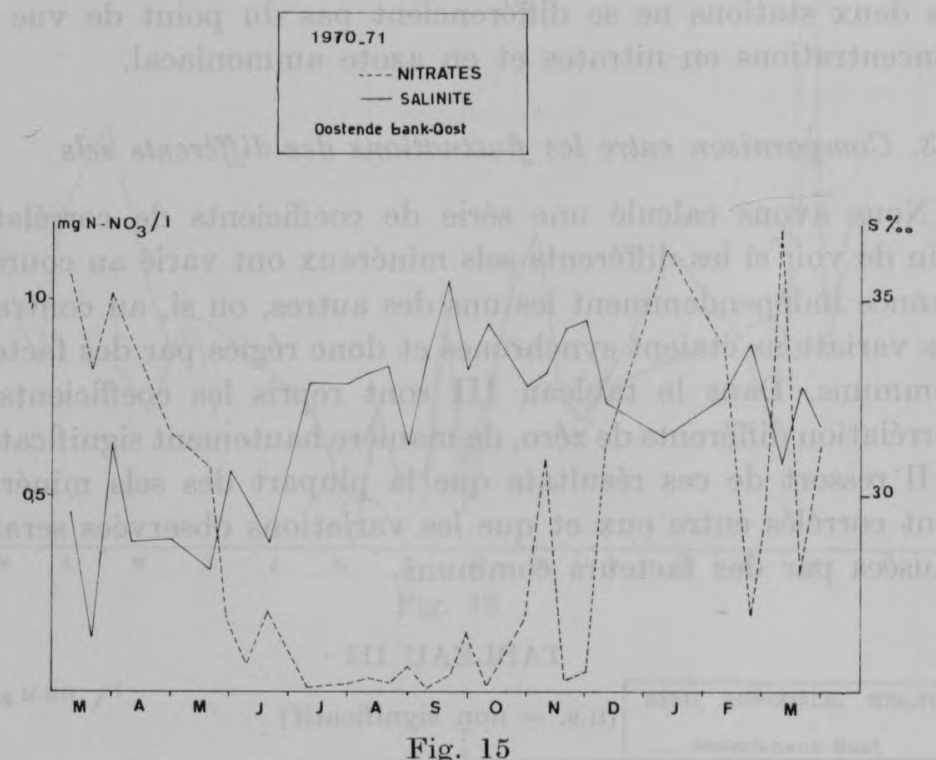

Des arrivées d'eaux douces, contenant de fortes quantités de sels minéraux à l'endroit de nos prélèvements, expliqueraient dès lors l'allure en "dents de scie" des courbes : les maxima seraient conséquents à des apports d'eaux douces et côtières, les minima à des arrivées d'eaux du large.

Les données bibliographiques concernant les teneurs en sels minéraux dans la Manche et la Mer du Nord sont difficilement comparables aux nôtres.

En effet, la fréquence des prélèvements varie d'un travail à l'autre. Certains auteurs, par exemple, ont fait plusieurs dosages par mois et publient des moyennes mensuelles s'étalant sur trois ans (L. Van Meel, 1957 ; F. A. Armstrong \& H. W. HarVEY, 1950). D'autres ont balayé une surface plus ou moins grande à certaines périodes de l'année (K. Kalle, 1953 ; G. Weichart, 1970 ; Programme C.I.P.S., 1971 et 1972). De plus, les chiffres cités dans la littérature concernent des années 
antérieures ou ultérieures à celles de nos prélèvements et les moyennes pourraient varier très fort d'une année à l'autre.

Il est done pratiquement impossible d'obtenir à partir de ces données bibliographiques des moyennes et des résultats statistiquement comparables aux nôtres.

Nous pouvons cependant relever quelques caractéristiques générales et descriptives concernant la distribution des sels minéraux et leurs variations saisonnières.

R. Johnston et P. G. W. Jones (1965) constatent que, comparativement à l'ensemble de la Mer du Nord, le Sud est très riche en éléments nutritifs. Cette richesse proviendrait des grands fleuves, Rhin-Meuse-Escaut, (dont le débit atteint $75 \mathrm{~km}^{3} / \mathrm{an}$ ) qui charrient les eaux usées provenant des villes et des industries et drainent les campagnes avoisinantes.

K. KalLE (1953) distingue quatre masses d'eau dans le sud de la Mer du Nord (figure 16). Les eaux côtières belges sont situées entre la zone 1 caractérisée par des eaux claires à haute salinité et pauvres en sels nutritifs, et la zone 4, riche en sels nutritifs, à faible salinité et forte turbidité. Les eaux littorales belges auraient peut-être des caractéristiques transitoires qu'il serait intéressant de vérifier par des recherches simultanées à plusieurs stations.

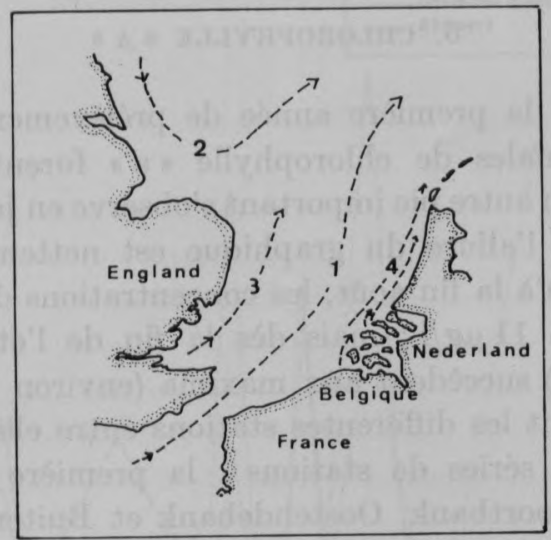

Fig. 16. - Principales masses d'eau dans le Sud de la Mer du Nord (d'après K. KALLE, 1953). 
Il y a peu de références sur les variations saisonnières des sels minéraux dans la partie sud de la Mer du Nord. D'après les cartes de R. Johnston et P. G. W. Jones (1965), les concentrations maximales en éléments nutritifs au large de la côte belge (phosphates, nitrates, silicates) s'observent à la fin de l'hiver et au début du printemps ; un premier minimum se rencontre en juillet-août, un second en novembre-décembre. L. VAN Meel (1957) écrit que "la moyenne de trois années (1951-1953) donne un maximum de nitrates en janvier avec $363,3 \mu \mathrm{g} N-\mathrm{NO}_{3} / 1$ et un minimum en septembre avec $95,8 \mu \mathrm{g} \mathrm{N}-\mathrm{NO}_{3} / 1 »$. C'est également en hiver, que nous avons observé les plus hautes concentrations en sels minéraux.

Enfin, I. Elskens (1971) rapporte que le phosphore total, les nitrates et les nitrites se diluent vers le large ; aux mailles 01,08 et $07 \mathrm{du}$ réseau expérimental C.I.P.S., les plus proches de nos stations de prélèvements ( $\mathrm{cfr}$. fig. 1) la concentration moyenne des nitrates n'excède pas $140 \mu \mathrm{g} \mathrm{N} / 1$ de juin à août 1971 ; celle des nitrites est comprise entre 1,5 et $9 \mu \mathrm{g} \mathrm{N} / 1$; en septembre, il observe une diminution des nitrates et des nitrites par rapport à l'été. Globalement, ces résultats concordent avec les nôtres. L'auteur rapporte également que des déversements volontaires et irréguliers sont effectués à proximité des mailles 07 et 08 et y entraîneraient des anomalies locales.

\section{CHLOROPHYLLE "A 》}

Au cours de la première année de prélèvement, les concentrations maximales de chlorophylle "a " furent atteintes au mois d'août. Un autre pic important s'observe en janvier (fig. 17). En 1970-1971, l'allure du graphique est nettement différente (fig. 18) : jusqu'à la fin août, les concentrations de chlorophylle n'excèdent pas $11 \mu \mathrm{g} / \mathrm{l}$; mais dès la fin de l'été, les minima (environ $5 \mu \mathrm{g} / \mathrm{l}$ ) succèdent aux maxima (environ $18 \mu \mathrm{g} / \mathrm{l}$ ).

En comparant les différentes stations entre elles, nous avons distingué deux séries de stations : la première comprend les bouées Nieuwpoortbank, Oostendebank et Buitenstroombank : situées près des côtes, elles se caractérisent par des hautes teneurs en chlorophylle. Les stations "off-shore " Oost-Dijck et 


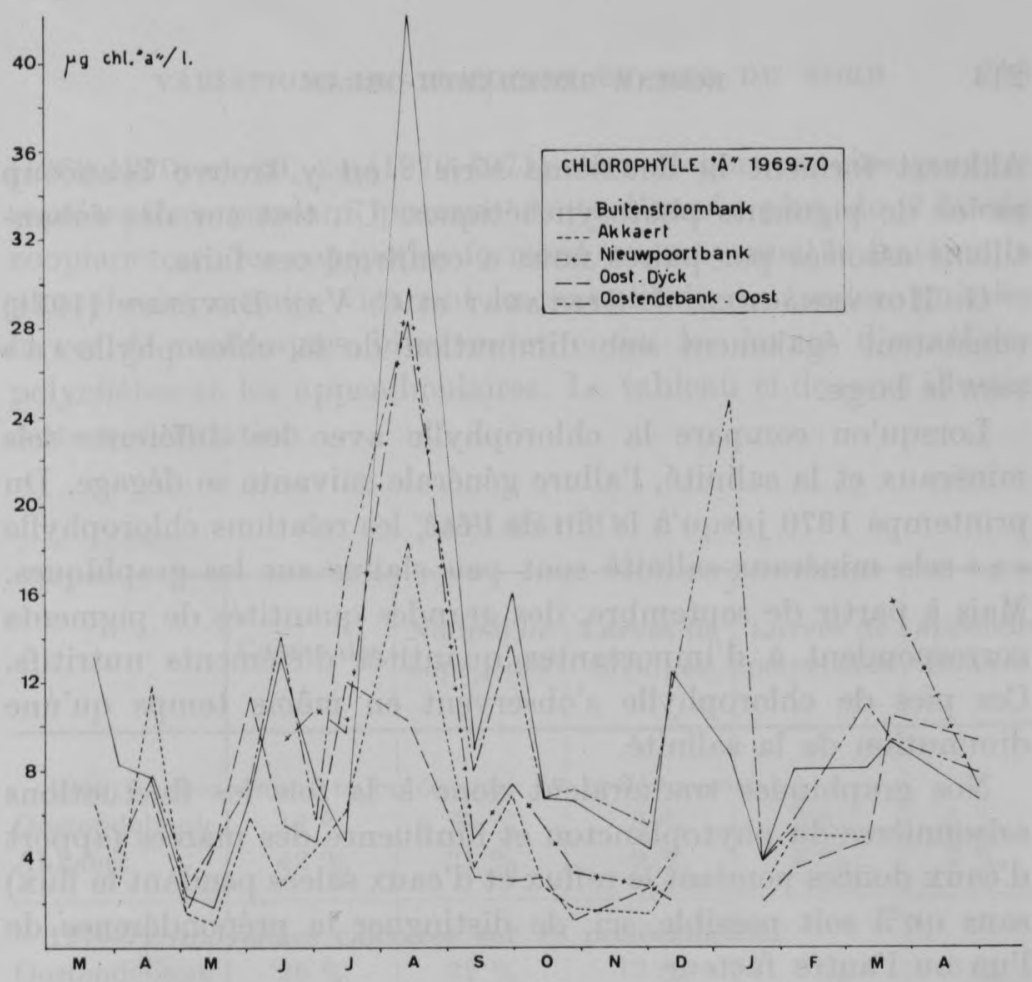

Fig. 17

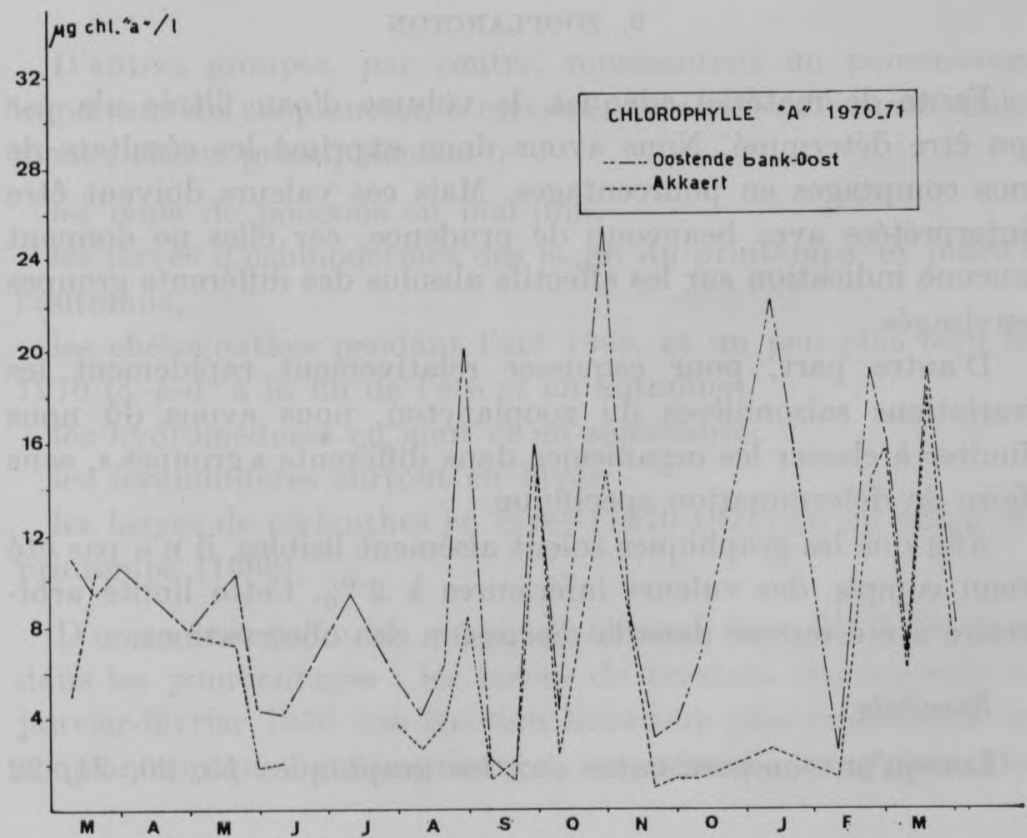

Fig. 18 
Akkaert forment la deuxième série : on y trouve beaucoup moins de pigments photosynthétiques. Un test sur des échantillons associés par paires nous a confirmé ces faits.

G. Houvenaghel, J. Steyaert et C. Van Beveren (1971) constatent également une diminution de la chlorophylle "a " vers le large.

Lorsqu'on compare la chlorophylle avec les différents sels minéraux et la salinité, l'allure générale suivante se dégage. Du printemps 1970 jusqu'à la fin de l'été, les relations chlorophylle « a »-sels minéraux-salinité sont peu claires sur les graphiques. Mais à partir de septembre, des grandes quantités de pigments correspondent à d'importantes quantités d'éléments nutritifs. Ces pics de chlorophylle s'observent en même temps qu'une diminution de la salinité.

Nos graphiques traduiraient done à la fois les fluctuations saisonnières du phytoplancton et l'influence des marées (apport d'eaux douces pendant le reflux et d'eaux salées pendant le flux) sans qu'il soit possible, ici, de distinguer la prépondérence de l'un ou l'autre facteur.

\section{ZOOPLANCTON}

Faute de matériel adéquat, le volume d'eau filtrée n'a pas pu être déterminé. Nous avons donc exprimé les résultats de nos comptages en pourcentages. Mais ces valeurs doivent être interprétées avec beaucoup de prudence, car elles ne donnent aucune indication sur les effectifs absolus des différents groupes envisagés.

D'autre part, pour esquisser relativement rapidement les variations saisonnières du zooplancton, nous avons dû nous limiter à classer les organismes dans différents "groupes », sans faire de détermination spécifique.

Afin que les graphiques soient aisément lisibles, il n'a pas été tenu compte des valeurs inférieures à $2 \%$. Cette limite arbitraire a été reprise dans la discussion des observations.

\section{Résultats}

Lorsqu'on compare entre eux les graphiques 19, 20, 21, 22 
(1969-1970) et 23,24 (1970-1971) on voit que certains groupes représentent pendant presque toute l'année plus de $2 \%$ du zooplancton : les copépodes forment, en moyenne, la fraction la plus élevée, ensuite viennent les nauplii de copépodes, puis les larves de mollusques bivalves et enfin, les larves d'annélides polychètes et les appendiculaires. Le tableau ci-dessous illustre cette constatation.

TABLEAU IV

\begin{tabular}{|c|c|c|c|c|c|}
\hline & Copépodes & $\begin{array}{l}\text { Nauplii de } \\
\text { copépodes }\end{array}$ & $\begin{array}{c}\text { Larves de } \\
\text { bivalves }\end{array}$ & $\begin{array}{l}\text { Larves de } \\
\text { polychètes }\end{array}$ & $\begin{array}{l}\text { appendi- } \\
\text { culaires }\end{array}$ \\
\hline \multicolumn{6}{|c|}{ 1969-70 (moyennes calculées sur 19 prélèvements) } \\
\hline Oostendebank & $42 \%$ & $25 \%$ & $9 \%$ & $3 \%$ & $5 \%$ \\
\hline Akkaert & $42 \%$ & $31 \%$ & $9 \%$ & $5 \%$ & $2 \%$ \\
\hline \multicolumn{6}{|c|}{ 1970-71 (moyennes calculées sur $18 \mathrm{p}$} \\
\hline Oostendebank & $38 \%$ & $22 \%$ & $12 \%$ & $7 \%$ & $5 \%$ \\
\hline Akkaert & $44 \%$ & $22 \%$ & $8 \%$ & $4 \%$ & $8 \%$ \\
\hline
\end{tabular}

D'autres groupes, par contre, représentent un pourcentage important du zooplancton à certaines périodes de l'année seulement; citons principalement :

les œufs de poissons en mai-juin,

les larves d'échinodermes dès la fin du printemps, et jusqu'à l'automne,

les chétognathes pendant l'été 1969 , et un peu plus tard en 1970 (c.-à-d. à la fin de l'été et en automne),

les hydroméduses en août et en septembre,

les foraminifères surtout en hiver,

les larves de cérianthes en hiver (1970-1971) ou au début du printemps (1969).

D'une année à l'autre, aussi, on observe de fortes différences dans les pourcentages : les larves de bivalves représentent en janvier-février 1970 une fraction beaucoup plus importante du zooplancton que l'année suivante ; inversément, les cyphonautes 
de bryozoaires sont plus abondantes pendant l'hiver 1970-1971 qu'en 1969-1970; les 3-4\% de nématodes observés en aoûtseptembre 1969 ne se retrouvent pas l'année suivante.

Dans l'ensemble, les variations observées à différentes stations au cours d'une même année sont assez homogènes. Les exceptions à cette synchronisation reflètent la distribution hétérogène de certaines espèces.

C'est le cas des larves de cirripèdes dont on trouve un pourcentage beaucoup moins élevé aux stations du large qu'aux stations côtières qui, elles sont proches des substrats solides où vivent les balanes (comme les brise-lames et les estacades).

A la mi-juillet 1969, la station Oostendebank présente $25 \%$ de rotifères. Peut-être sont-ils des indicateurs d'une arrivée d'eau saumâtre à cet endroit, mais seule une détermination spécifique pourrait répondre à cette question.

Légende

\begin{tabular}{|c|c|}
\hline & Foraminifères \\
\hline & Coelentérés \\
\hline & Larves d'annélides polychètes \\
\hline & Cladocères \\
\hline,++ & Copépodes \\
\hline & Nauplii de copépodes \\
\hline 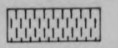 & Larves de cirripèdes \\
\hline & Larves de décapodes \\
\hline & Chétognathes \\
\hline$\beta$ & Nématodes \\
\hline 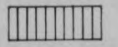 & Rotifères \\
\hline 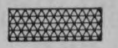 & Cyphonautes de bryozoaires \\
\hline & Larves d'échinodermes \\
\hline & Larves de mollusques bivalves \\
\hline & Appendiculaires \\
\hline$\because \because \because \cdots$ & Eufs et larves de poissons \\
\hline & Divers \\
\hline
\end{tabular}


Oost - Dijck 1969-1970

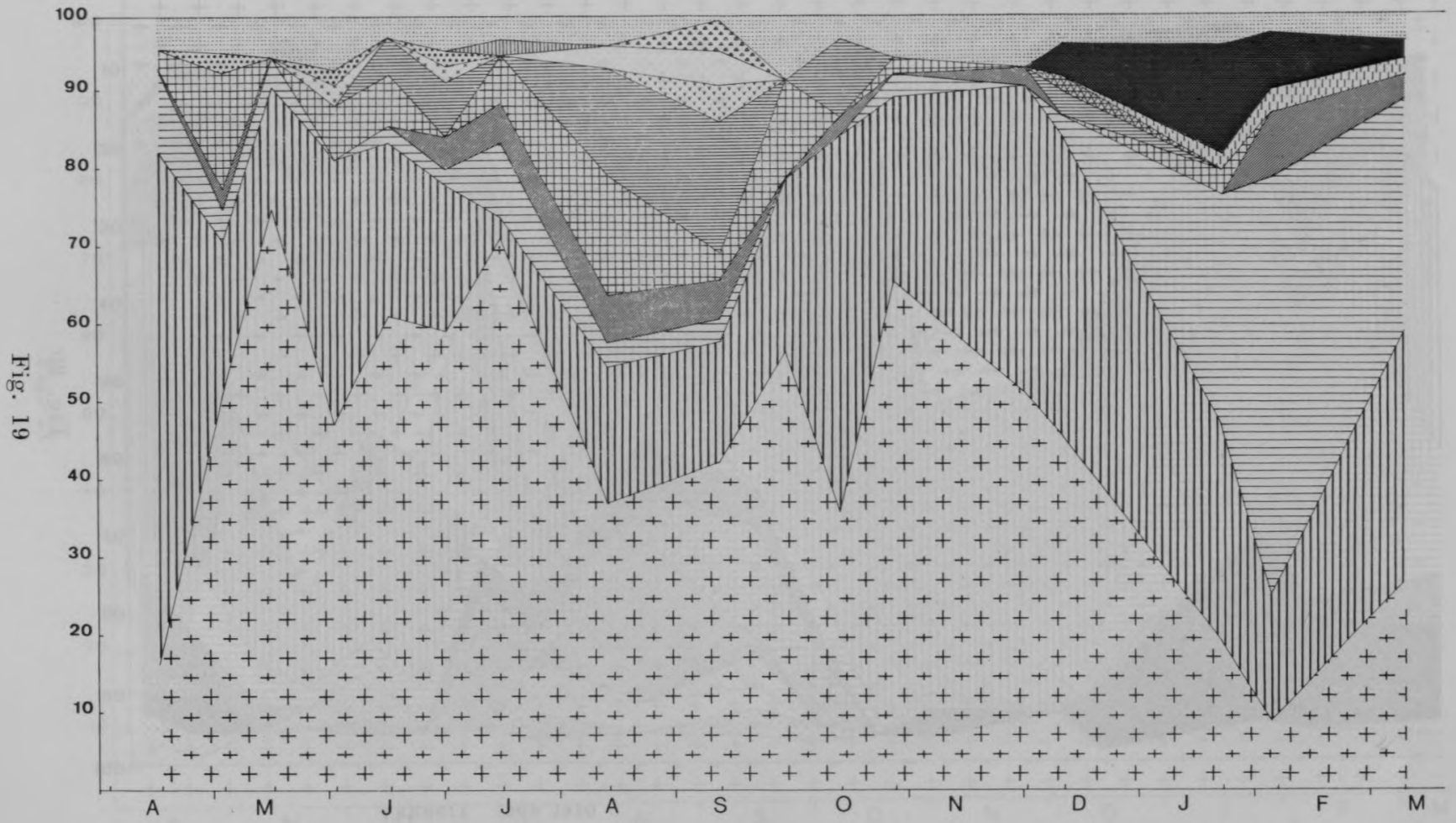




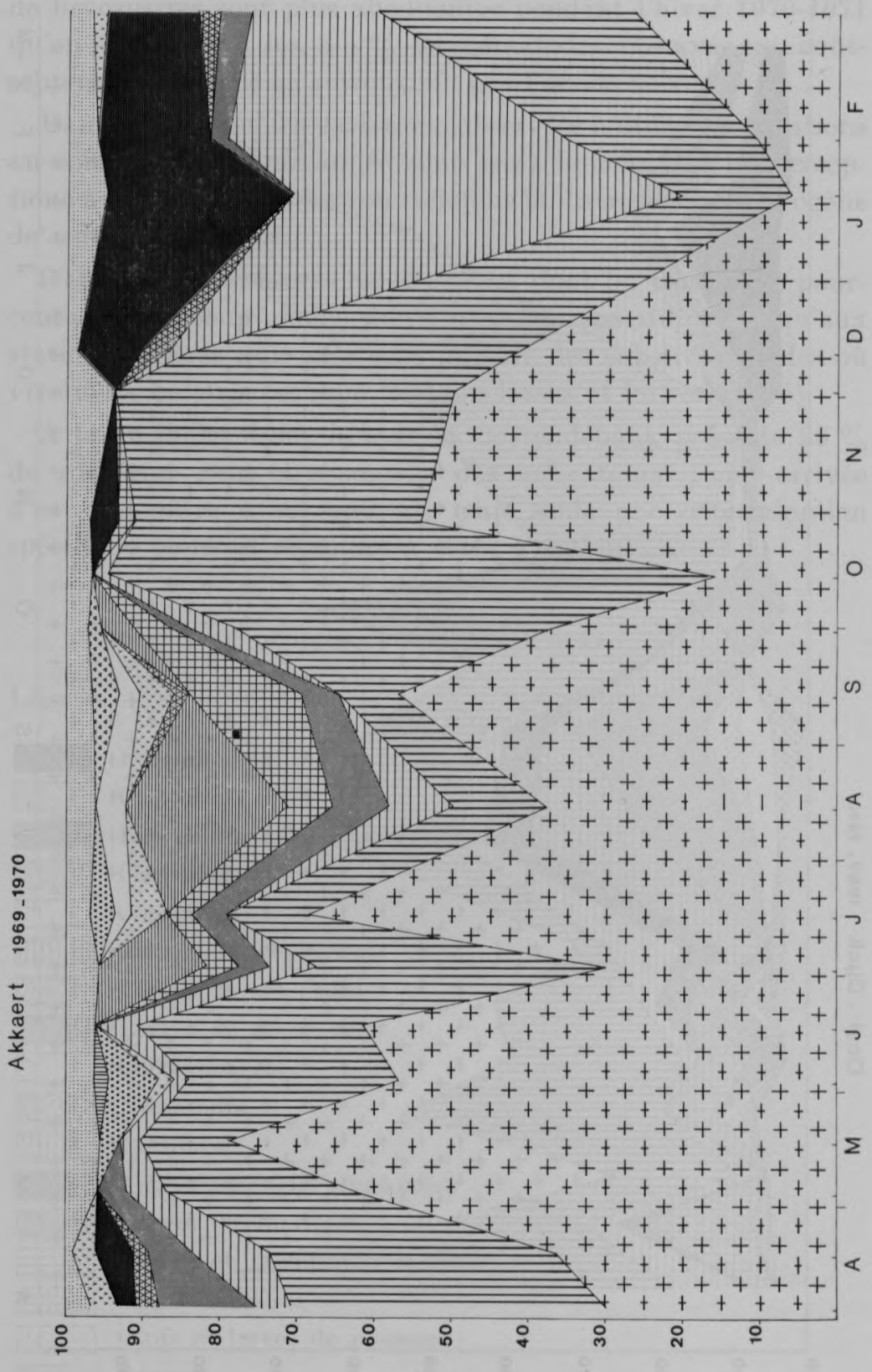

Fig. 20 


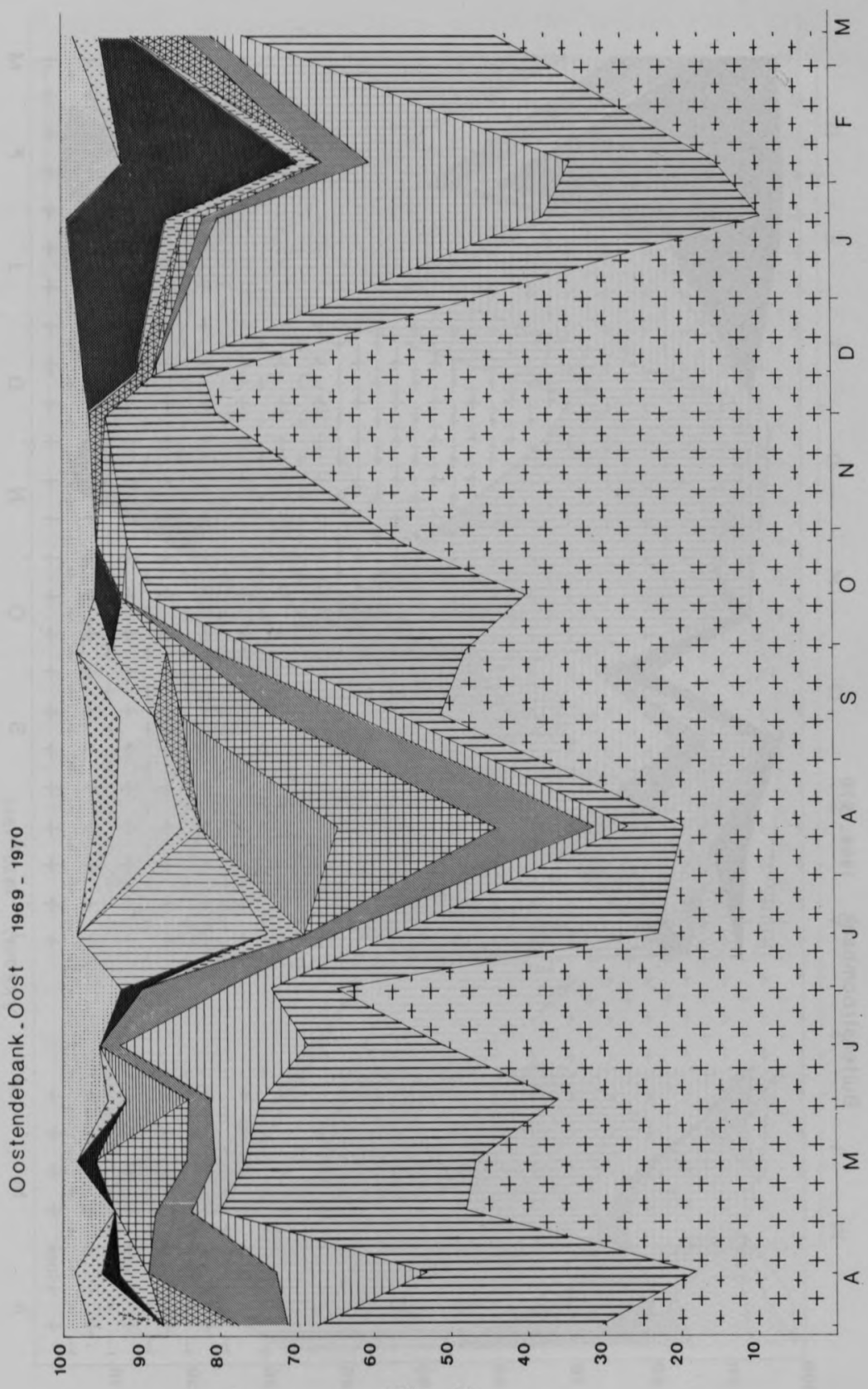

Fig. 21 


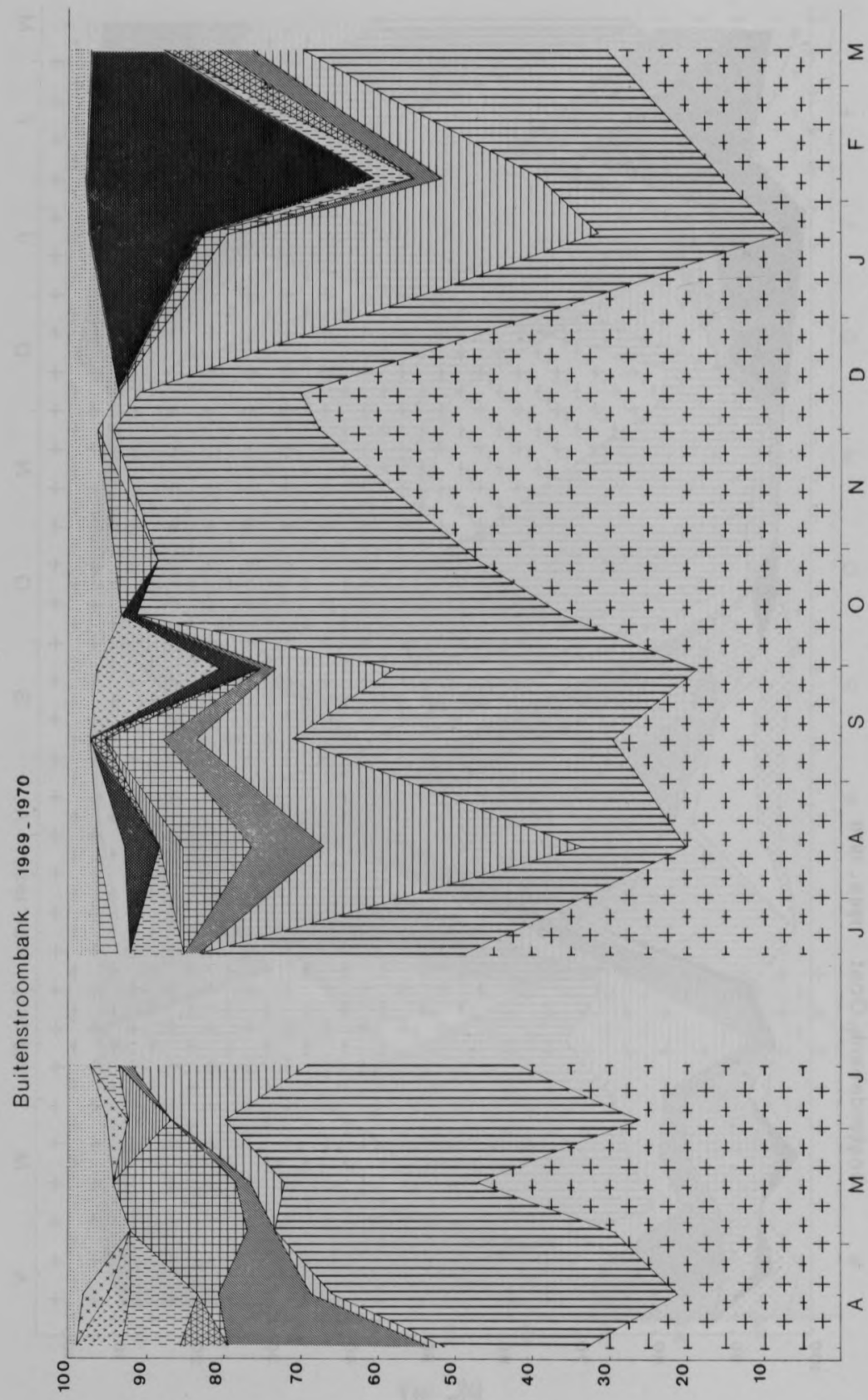

Fig. 22 


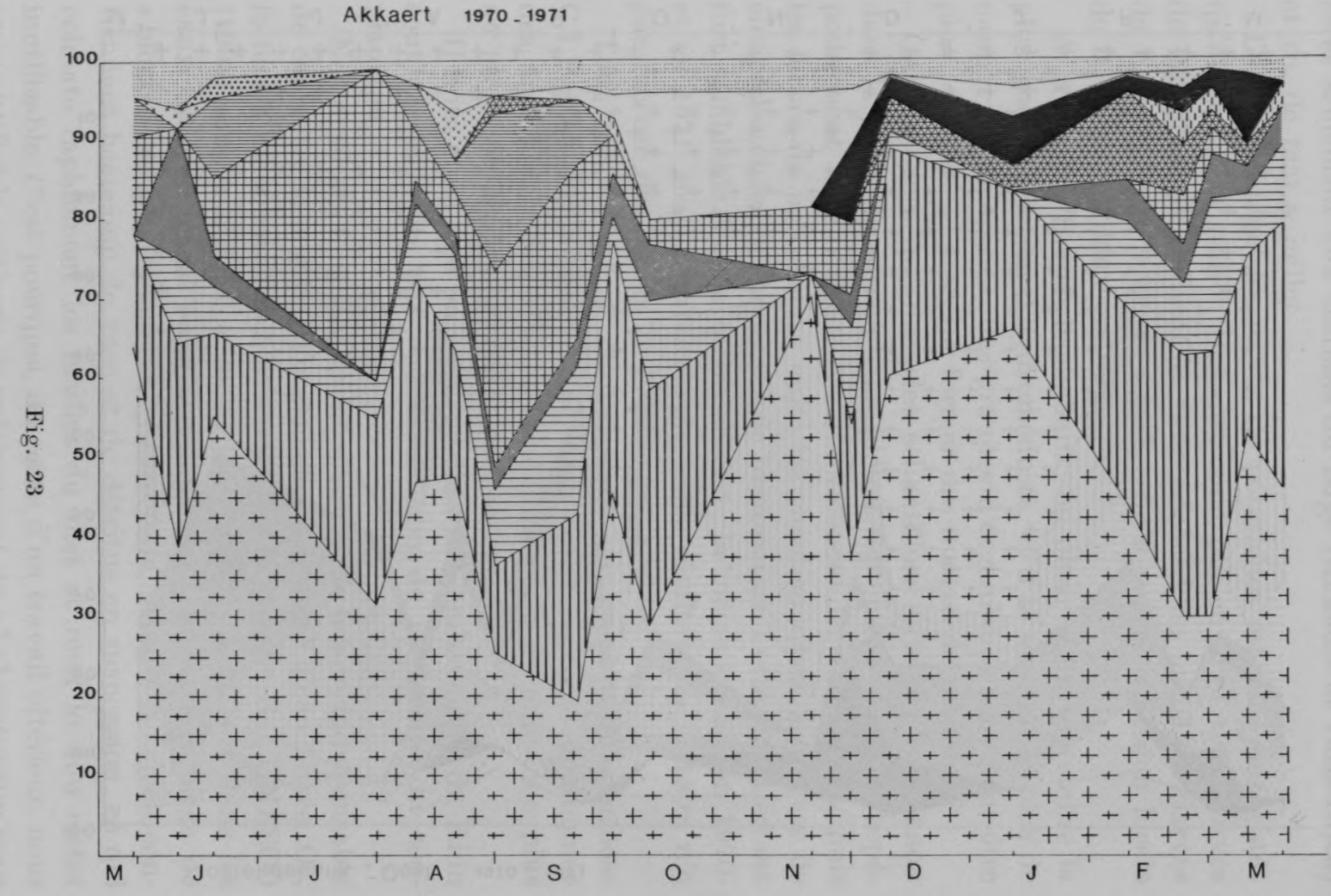




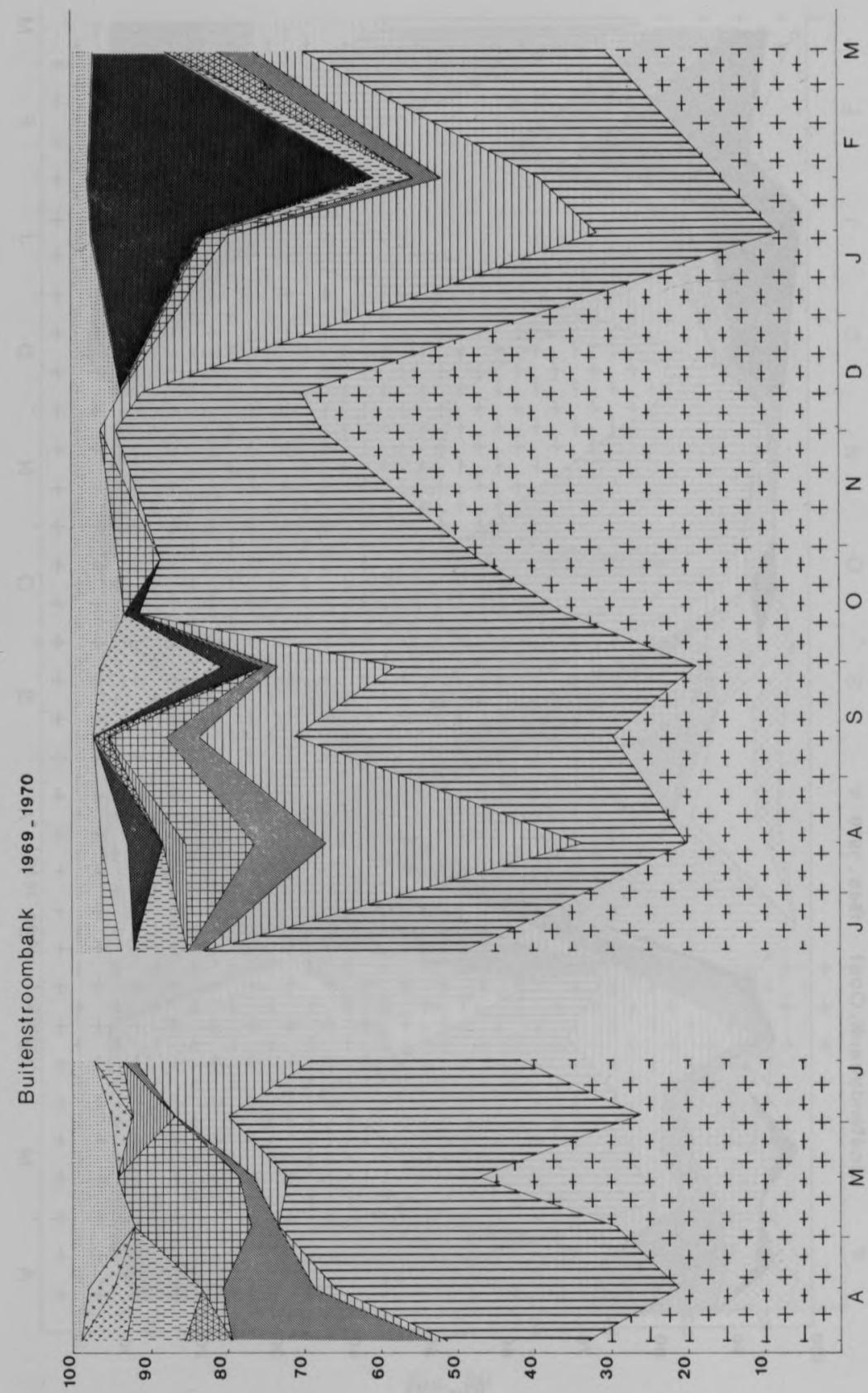

Fig. 22 


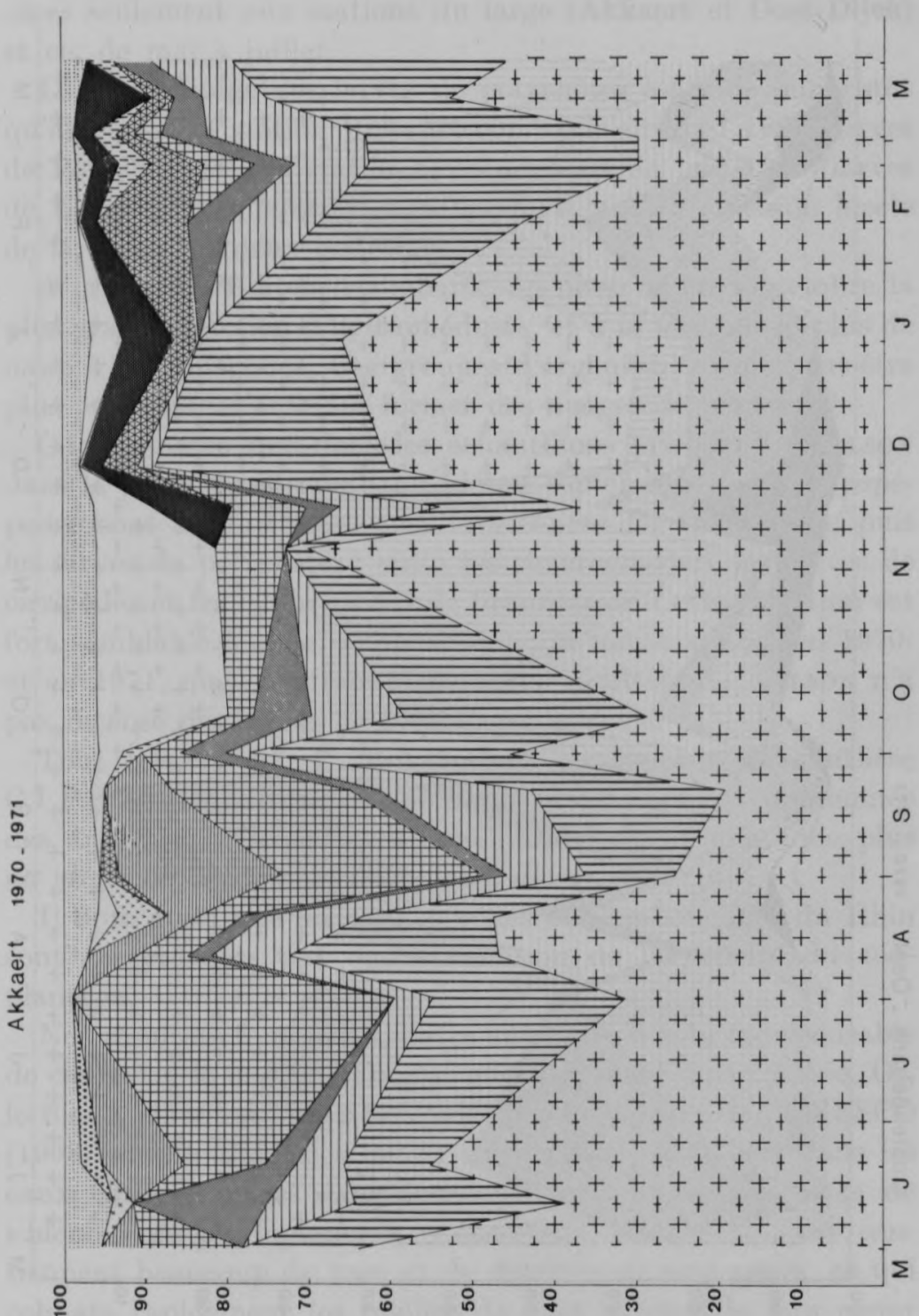

Fig. 23 


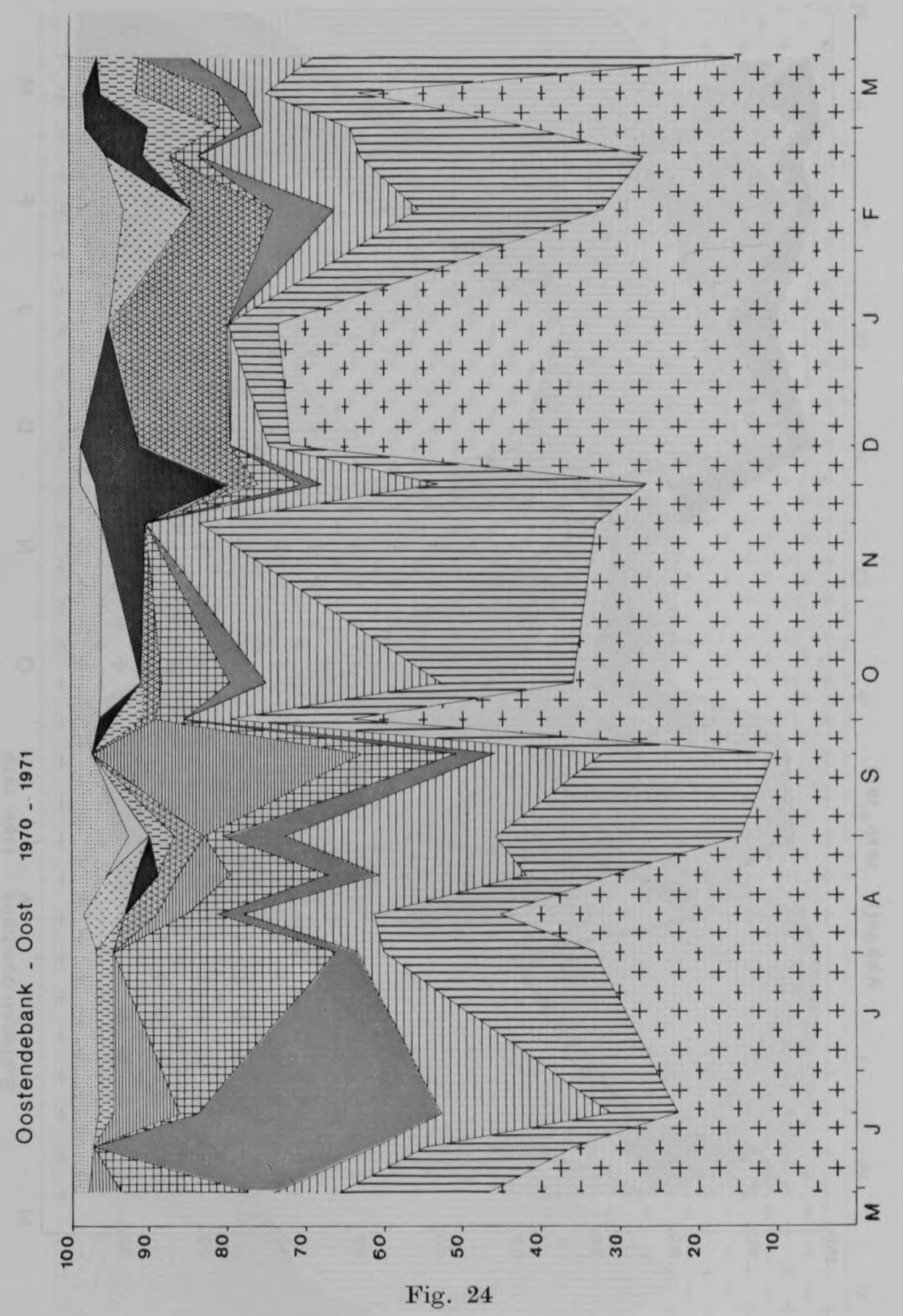


Pendant les deux années successives, on retrouve des cladocères seulement aux stations du large (Akkaert et Oost-Dijck) et ce, de mai à juillet.

Le pourcentage des larves de polychètes est plus important qu'ailleurs à la station Buitenstroombank en avril 1969 (larves de Polydora) et à la station Oostendebank en juin 1970 (larves de Lanice). Cela pourrait signifier qu'il y a eu émission locale de larves à ces deux endroits.

A la station Buitenstroombank, on observe fin septembre la plus grande fraction d'hydroméduses, et à la mi-août le plus de nauplii de copépodes. Ces groupes d'organismes sont peut-être plus aptes que d'autres à former des nuages de plancton?

Citons encore que dans les échantillons pris par G. GILson dans la passe du Stroombank (début septembre 1907) les copépodes sont majoritaires; ensuite viennent les Oikopleura, puis les larves de polychètes; enfin les chaetognathes, les larves de cirripèdes et les cyphonautes de bryozoaires. Cette situation est fort semblable à celle qu'on observe, la même saison, en 1970et en 1971, mais contrairement à nos résultats, G. GILson n'a pas recensé des larves de bivalves en surface.

Tout récemment, $P$. PoLk, dans un rapport de synthèse C.I.P.S. (1972) distingue une zone côtière à forte dominance des annélides, cirripèdes et lamellibranches, et une zone plus au large, ou prédomine Oikopleura dioica.

Il note aussi que les eaux proches de l'embouchure du Rhin sont caractérisées par une diminution de la densité du zooplancton.

Nos résultats font sentir, entre autres, le besoin indispensable de connaître le nombre d'organismes par unité d'eau filtrée. Or, le filet à plancton WP-2 préconisé par le rapport de l'UNESCO (1968) couplé àun "flow-meter » est difficile à utiliser dans les eaux côtières belges; car ces eaux sont souvent le siège de "bloom » de phytoplancton (Phaeocystis, Noctiluca ...) et contiennent beaucoup de vase et de détritus en suspension, ce qui colmate rapidement les mailles du filet et rend le flow-meter inutilisable. C'est pourquoi, au cours d'un travail ultérieur, nous avons utilisé la méthode de prélèvement du «Laboratorium voor Ekologie en Systematiek " (Vrije Universiteit Brussel) du 
Pr. Polk : il s'agit de prélever à la pompe ou au seau une cinquantaine de litres d'eau de mer et de les filtrer au travers d'un filet à mailles fines. Cette méthode nous a permis une collecte et une numération des organismes très satisfaisante.

\section{CONCLUSIONS GÉNÉRALES}

Avant de tirer des conclusions de ces quelques données, il faut d'abord souligner les limites de ce travail.

En effet, le nombre de stations étudiées (2 ou 5) est insuffisant pour caractériser la distribution de nos eaux côtières.

De même, la fréquence des prélèvements (tous les 15 jours environ) ne révèle pas les fortes variations qui pourraient se produire éventuellement, entre deux prélèvements.

Enfin, les analyses effectuées s'étendent sur deux ans maximum, laps de temps trop court pour fournir un schéma représentatif des interactions existant entre les domaines physiques, chimiques et biologiques.

L'ensemble des résultats obtenus permet cependant d'acquérir des données générales sur les conditions hydrologiques et physico-chimiques ainsi que sur les variations du plancton au large d'Oostende et de Nieuwpoort.

Les eaux littorales belges sont peu profondes et bien brassées lors de tempêtes, ce qui remet régulièrement les sédiments en suspension.

Les concentrations en sels minéraux sont élevées et les salinités relativement basses lorsqu'on les compare à celles de la Manche ou du centre de la Mer du Nord. C'est en hiver qu'on observe les plus fortes quantités d'éléments nutritifs.

En ce qui concerne les pigments photosynthétiques, les deux années étudiées ont peu de caractères communs. Nous n'avons pas retrouvé l'important pic de printemps et le petit pic d'automne typique des eaux tempérées nordiques (H. B. Moore, 1966). Les grandes quantités de pigments observées en plein hiver pourraient être dûes à des organismes morts ; en effet, d'après G. Houvenaghel, J. Steyaert et C. Van Beveren (1971) les concentrations en chlorophylle "a " et phaeophytine " a » sont élevées près des côtes et diminuent vers le large ; 
mais le rapport cellules vivantes/cellules mortes augmente avec l'éloignement de la côte et de l'estuaire de l'Escaut.

Des comparaisons entre les différentes stations étudiées nous permettent de dire qu'elles suivent les mêmes courbes de fluctuation et nous suggèrent que la dessalure, la concentration en éléments nutritifs et la quantité de phytoplancton sont plus accusées aux trois stations côtières qu'aux deux stations du large.

Il semble que la plupart des phénomènes relevés ci-dessus sont dûs à des apports d'eaux douces côtières qui perturbent plus ou moins fort les stations étudiées suivant leur éloignement de la côte. Mais nous avons trop peu de données pour distinguer si e'est le réseau des canaux de la côte belge ou le delta RhinMeuse-Escaut qui est à l'origine de ces caractéristiques.

Nous espérons qu'un programme comme celui de la C.I.P.S., comprenant une étude approfondie des courants de marée et de la salinité, menée en même temps que des prélèvements continus pendant une certaine période, sur une aire importante, ainsi qu'une analyse quantitative et qualitative du plancton et le dosage des phaeopigments, éclairera les résultats que nous avons obtenus.

Je tiens en terminant à remercier Mr le Prof. J. BoutLuon qui a tout mis en œuvre pour me permettre d'effectuer ce travail. Je remercie également $\mathrm{M}^{\mathrm{r}}$ et $\mathrm{M}^{\mathrm{m}} \mathrm{M}$. STEyaERT pour leurs précieux conseils, ainsi que Mme Defrise-Gussenhoven, $\mathbf{M}^{\mathrm{r}}$ le Prof. P. Polk et tous les membres du Laboratoire d'Anatomie Comparée qui m'ont aidée.

Ce travail a été rendu possible grâce à la collaboration de la Force Navale et du Ministère de l'Éducation Nationale et de la Culture Française et grâce à l'hospitalité reçue à la "Zeevisserijschool J. BAuWEns » d'Oostende.

Enfin, je remercie mon père $P$. Orban et les membres du "Yacht Club Nieuwpoort " qui ont coopéré avec enthousiasme à de nombreux prélèvements en mer.

\section{RÉFÉRENCES BIBLIOGRAPHIQUES}

Armstrong, F. A. \& H. W. Harvey (1950). - The cycle of the phosphorus in the waters of the English Channel. J. mar. biol. Ass. U.K., 29, $145-162$. 
Atlas de Température et de Salinité de l'eau de surface de la Mer du Nord et de la Manche (1933). Conseil perm. int. Explor. Mer, Copenhagen.

Chоw, T. J. \& M. S. Johnstone (1962). — Determinaiton of nitrate in sea-water. Analytica chim. Acta, 27, 441-446.

C.I.P.S. (1971). - Programme national belge sur l'environnement physique et biologique (C.I.P.S.). Modèle mathématique. Rapport de synthèse. Journées d'études des 24-25 novembre 71, 1.

C.I.P.S. (1972). - Programme national belge sur l'environnement physique et biologique (C.I.P.S.). Modèle mathématique. Rapport de synthèse. Journées d'études des 6-7-8 novembre 72, 2.

De Pauw, N. (1969). - Contribution à l'étude du plancton dans le port d'Ostende. Biol. Jaarb., 37, 186-262.

ELSKEns, I. (1971). - Nutrients et params̀tres biologiques associés. Rapport de synthèse (C.I.P.S.), 1, 96-122.

Gerets, M. C. (1970). - Esquisse de l'écologie du chenal de Nieuport. Mémoire de licence, Université Libre de Bruxelles.

GiLson, G. (1900). - Exploration sur les côtes de Belgique en 1899. Mém. Mus. Roy. Nat. Belg., 1.

GiLson, G. (1907). - Exploration de la mer sur les côtes de Belgique Recherches sur le milieu marin et ses variatons au voisinage de la. côte belge. Mém. Mus. Roy. Hist. Nat. Belg., 4, 1-87.

Gilson, G. (1924). - Exploration de la mer sur les côtes de Belgique. Recherches sur la dérive dans la mer du Nord. Mém. Mus. Roy. Hist. Nat. Belg., 35.

Houvenagel, G., Steyaert, J. \& C. Van Beveren (1971). - First trophic level - Analysis of photosynthetic pigments and phytoplancton. Rapport de synthèse (C.I.P.S.), 1, 151-161.

Johnston, R. \& P. G. W. Jones (1965). - Inorganic nutrients in the North sea. Serial Atlas of the Marine Environment, 11. American Geographical Society, New-York.

KaLLe, K. (1953). - Der Einfluss des Englischen Küstenwassers auf den Chemismus der Wasserkörper in der Südlichen Nordsee. Ber. dt. wiss Kommn Meeresforsch., 13 (2), 130-135.

Lefìvere, S., Leloup, E. \& L. VAN Meel (1956). - Observations biologiques dans le port d'Ostende. Mém. Inst. Roy. Nat. Belg., 133, 1-157.

Leloup, E. \& B. Konietzko (1956). - Recherches biologiques sur les 
eaux saumâtres du Bas-Escaut. Mém. Inst. Roy. Sci. Nat. Belg., 132, 1-100.

Leloup, E. (1966). - Observations sur la dérive des courants au large de la côte belge au moyen de flotteurs de fond. Bull. Inst. Roy. Sci. Nat. Belg., 42, 20.

Moore, H. B. (1966). - Marine Ecology. John Wiley, New-York, U.S.A.

Ріснот, G. (1971). - Courants. Rapport de synthèse (C.I.P.S.), 1, 28-34.

Polk, P. (1972). - Le plancton. Rapport de synthèse (C.I.P.S.), 2, 353-369.

Poncelet \& Martin (1948). - Extraits de "Esquisses climatographiques » de la Belgique. Publication de l'Institut Royal Météorologique de Belgique, Bruxelles.

Ronday, C., Runfolla, Y. \& Y. Adam (1972). - Modèles hydrodynamiques. Rapport de synthèse (C.I.P.S.), 2, 35-137.

Symposium on the classification of Brackish Waters. Final resolution (1959). Archo Oceanogr. Limnol., 11 (suppl.), 243-245.

Strickland, J. D. H. \& J. R. Parsons (1968). - A practical handbook of sea-water analysis. Bull. Fish. Res. Bd. Can., 167, 1-311.

UNESCO (1969). - Determination of photosynthetic pigments in sea-water. Monographs on oceanographic methodology, 1, 69 p. UNES$\mathrm{CO}$, Paris.

UNESCO (1968). - Zooplankton sampling. Monographs on oceanographic methodology, 2, 174 p. UNESCO, Paris.

VAN MEeL, L. (1957). - Le milieu marin au bateau-phare "West-Hinder"• Période 1951-55. Bull. Inst. Roy. Sci. Nat. Belg., 33 (4), 1-33.

Weichart, G. (1970). - Regiestrierung des Phosphat-Gehalts im Oberflächenwasser der Nordsee und Armelkanals. Ber. dt. Kommn Meeresforsch., 21, 410-419. 